\title{
DISCRETIZATION OF LINEAR PROBLEMS IN BANACH SPACES: RESIDUAL MINIMIZATION, NONLINEAR PETROV-GALERKIN, AND MONOTONE MIXED METHODS*
}

\author{
IGNACIO MUGA ${ }^{\dagger}$ AND KRISTOFFER G. VAN DER ZEE
}

Dedicated to Leszek Demkowicz and J. Tinsley Oden

\begin{abstract}
This work presents a comprehensive discretization theory for abstract linear operator equations in Banach spaces. The fundamental starting point of the theory is the idea of residual minimization in dual norms, and its inexact version using discrete dual norms. It is shown that this development, in the case of strictly-convex reflexive Banach spaces with strictly-convex dual, gives rise to a class of nonlinear Petrov-Galerkin methods and, equivalently, abstract mixed methods with monotone nonlinearity. Under the Fortin condition, we prove discrete stability and quasioptimal convergence of the abstract inexact method, with constants depending on the geometry of the underlying Banach spaces. As part of our analysis, we obtain new bounds for best-approximation projectors. The theory generalizes and extends the classical Petrov-Galerkin method as well as existing residual-minimization approaches, such as the discontinuous Petrov-Galerkin method.
\end{abstract}

Key words. Operators in Banach spaces, Residual minimization, Petrov-Galerkin discretization, Error analysis, Quasi-optimality, Duality mapping, Best approximation, Geometric constants

AMS subject classifications. 41A65, 65J05, 46B20, 65N12, 65N15

1. Introduction. The discontinuous Petrov-Galerkin (DPG) methodology developed by Demkowicz and Gopalakrishnan, and, more generally, minimal-residual (MinRES) formulations with residual measured in a dual norm, have attracted significant attention in the numerical analysis literature [19, 20,2], owing to their conceptual simplicity and striking stability properties. In this paper we provide an abstract stability and convergence analysis of the (practical) inexact version within Banach space settings. Our analysis extends the Hilbert-space analysis by Gopalakrishnan \& Qiu [26], and thereby opens up a convergence theory for the MinRES discretization of partial differential equations (PDEs) in non-standard non-Hilbert settings.

1.1. MinRes methods in Banach spaces. For our analysis, we consider the abstract problem

$$
\left\{\begin{array}{c}
\text { Find } u \in \mathbb{U}: \\
B u=f \quad \text { in } \mathbb{V}^{*}
\end{array}\right.
$$

where $\mathbb{U}$ and $\mathbb{V}$ are infinite-dimensional Banach spaces and the data $f$ is a given element in the dual space $\mathbb{V}^{*}$. The operator $B: \mathbb{U} \rightarrow \mathbb{V}^{*}$ is a continuous, boundedbelow linear operator, that is, there is a continuity constant $M_{B}>0$ and bounded-

*Submitted to the editors DATE.

Funding: The work by IM was done in the framework of Chilean FONDECYT research project \#1160774. IM was also partially supported by European Union's Horizon 2020 research and innovation program under the Marie Sklodowska-Curie grant agreements No 644202 and No 777778 . KvdZ is grateful to the support provided by the Royal Society International Exchanges Scheme / Kan Tong Po Visiting Fellowship Programme, and by the above-mentioned FONDECYT project.

${ }^{\dagger}$ Instituto de Matemáticas, Pontificia Universidad Católica de Valparaíso, Chile (ignacio.muga@pucv.cl).

${ }^{\ddagger}$ School of Mathematical Sciences, University of Nottingham, UK (kg.vanderzee@nottingham.ac.uk). 
below constant $\gamma_{B}>0$ such that

$$
\gamma_{B}\|w\|_{\mathbb{U}} \leq\|B w\|_{\mathbb{V} *} \leq M_{B}\|w\|_{\mathbb{U}}, \quad \forall w \in \mathbb{U} .
$$

We shall assume throughout this paper the existence of a unique solution. ${ }^{1}$ Note that problem (1.1) is equivalent to the variational statement

$$
\langle B u, v\rangle_{\mathbb{V}^{*}, \mathbb{V}}=\langle f, v\rangle_{\mathbb{V}^{*}, \mathbb{V}} \quad \forall v \in \mathbb{V},
$$

commonly encountered in the weak formulation of PDEs, i.e., when $\langle B u, v\rangle_{\mathbb{V}^{*}, \mathbb{V}}=$ : $b(u, v)$ and $b: \mathbb{U} \times \mathbb{V} \rightarrow \mathbb{R}$ is a bilinear form.

Given a discrete (finite-dimensional) subspace $\mathbb{U}_{n} \subset \mathbb{U}$, the exact (or ideal) MinRes formulation for the above problem is: ${ }^{2}$

$$
\left\{\begin{array}{l}
\text { Find } u_{n} \in \mathbb{U}_{n}: \\
\quad u_{n}=\arg \min _{w_{n} \in \mathbb{U}_{n}}\left\|f-B w_{n}\right\|_{\mathbb{V}^{*}},
\end{array}\right.
$$

where the dual norm is given by

$$
\|g\|_{\mathbb{V}^{*}}=\sup _{v \in \mathbb{V} \backslash\{0\}} \frac{\langle g, v\rangle_{\mathbb{V}^{*}, \mathbb{V}}}{\|v\|_{\mathbb{V}}}, \quad \text { for any } g \in \mathbb{V}^{*} .
$$

This formulation is appealing for its stability and quasi-optimality without requiring additional conditions, which was proven by Guermond [27], who studied residual minimization abstractly in Banach spaces, and focussed on the case where the residual is in an $L^{p}$ space, for $1 \leq p<\infty$.

Although the MinRES formulation (1.3) is quasi-optimal, an essential complication is that the dual norm (1.4) may be non-computable in practice, because it requires the evaluation of a supremum over $\mathbb{V}$ that may be intractable. This is the case, for example, when $\mathbb{V}^{*}$ is a negative Sobolev space such as $\left[W_{0}^{1, p}(\Omega)\right]^{*}=: W^{-1, q}(\Omega)$, where $p^{-1}+q^{-1}=1,1 \leq p \leq \infty$, and $\Omega \subset \mathbb{R}^{d}$ is a bounded $d$-dimensional domain. Situations with non-computable dual norms are very common in weak formulations of PDEs and, therefore, these complications can not be neglected.

A natural replacement that makes such dual norms computationally tractable is obtained by restricting the supremum to discrete subspaces $\mathbb{V}_{m} \subset \mathbb{V}$. This idea leads to the following inexact MinRES problem:

$$
\left\{\begin{array}{l}
\text { Find } u_{n} \in \mathbb{U}_{n}: \\
u_{n}=\arg \min _{w_{n} \in \mathbb{U}_{n}}\left\|f-B w_{n}\right\|_{\left(\mathbb{V}_{m}\right)^{*}},
\end{array}\right.
$$

where the discrete dual norm is now given by ${ }^{3}$

$$
\|g\|_{\left(\mathbb{V}_{m}\right)^{*}}=\sup _{v_{m} \in \mathbb{V}_{m} \backslash\{0\}} \frac{\left\langle g, v_{m}\right\rangle_{\mathbb{V}^{*}, \mathbb{V}}}{\left\|v_{m}\right\|_{\mathbb{V}}}, \quad \text { for any } g \in \mathbb{V}^{*} .
$$

Note that a notation with a separate parametrization $(\cdot)_{m}$ is used to highlight the fact that $\mathbb{V}_{m}$ need not necessarily be related to $\mathbb{U}_{n}$.

\footnotetext{
${ }^{1}$ which is guaranteed provided $f \in \operatorname{Im} B$ or $\operatorname{Ker} B^{*}=\{0\}$ ( $B$ is surjective); see, e.g., [24, Appen$\operatorname{dix}$ A.2], [37, Section 5.17]. The smallest possible $M_{B}$ coincides with $\|B\|:=\sup _{w \in \mathbb{U} \backslash\{0\}}\|B w\|_{\mathbb{V}^{*}} /\|w\|_{\mathbb{U}}$ while the largest possible $\gamma_{B}$ coincides with $1 /\left\|B^{-1}\right\|$, where $B^{-1}: \operatorname{Im}(B) \rightarrow \mathbb{U}$.

${ }^{2}$ If $\mathbb{V}$ is a Hilbert space, residual minimization corresponds to the familiar least-squares minimization method [4]; otherwise it requires the minimization of a convex (non-quadratic) functional.

${ }^{3}$ Strictly speaking, the discrete dual norm is a norm on $\left(\mathbb{V}_{m}\right)^{*}$ and only a semi-norm on $\mathbb{V}^{*}$.
} 
1.2. Main results. The main objective of our work is to present equivalent formulations, prove the stability (uniform discrete well-posedness), and provide a quasi-optimal convergence analysis for the inexact MinRES discretization (1.5).

Most of our results are valid in the case that $\mathbb{V}$ is a reflexive Banach space such that $\mathbb{V}$ and $\mathbb{V}^{*}$ are strictly convex ${ }^{4}$, which we shall refer to as the reflexive smooth setting. This setting includes Hilbert spaces, but also important non-Hilbert spaces, since $L^{p}(\Omega)$ (as well as $p$-Sobolev spaces) for $p \in(1, \infty)$ are reflexive and strictly convex, however not so for $p=1$ and $p=\infty$ (see [14, Chapter II] and [7, Section 4.3]). We assume this special setting throughout the remainder of Section 1.

Indispensable in developing equivalent formulations is the duality mapping $J_{\mathbb{V}}$ : $\mathbb{V} \rightarrow \mathbb{V}^{*}$, which is a well-studied operator in nonlinear functional analysis that can be thought of as the extension to Banach spaces of the well-known Riesz map (which is a Hilbert-space construct). In the reflexive smooth setting, the duality mapping is a bijective monotone operator that is nonlinear in the non-Hilbert case. ${ }^{5}$

The main assumption in the analysis of stability and quasi-optimality, pertains to a compatibility requirement on the pair $\left(\mathbb{U}_{n}, \mathbb{V}_{m}\right)$. Analogous to the Hilbert-space case [26], this compatibility is stated in terms of Fortin's condition (involving a Fortin operator $\Pi: \mathbb{V} \rightarrow \mathbb{V}_{m}$, see Assumption 4.4 in Section 4.2), which is essentially a discrete inf-sup condition on $\left(\mathbb{U}_{n}, \mathbb{V}_{m}\right)[25]$.

Our main results and novel contributions are as follows:

- (Theorem 4.1) The discrete solution to the inexact MinRes problem (1.5) is equivalently characterized by the statement: ${ }^{6}$

$$
\left\{\begin{array}{l}
\text { Find } u_{n} \in \mathbb{U}_{n}: \\
\quad\left\langle\nu_{n}, J_{\mathbb{V}_{m}}^{-1}\left(f-B u_{n}\right)\right\rangle_{\mathbb{V}^{*}, \mathbb{V}}=0 \quad \forall \nu_{n} \in B \mathbb{U}_{n} \subset \mathbb{V}^{*},
\end{array}\right.
$$

which we refer to as an (inexact) nonlinear Petrov-Galerkin method. In turn, this is equivalent to a constrained-minimization formulation (or a saddle-point problem), which in mixed form reads:

$$
\left\{\begin{array}{rlrl}
\text { Find }\left(r_{m}, u_{n}\right) \in \mathbb{V}_{m} \times \mathbb{U}_{n}: & \\
\left\langle J_{\mathbb{V}}\left(r_{m}\right), v_{m}\right\rangle_{\mathbb{V}^{*}, \mathbb{V}}+\left\langle B u_{n}, v_{m}\right\rangle_{\mathbb{V}^{*}, \mathbb{V}} & =\left\langle f, v_{m}\right\rangle_{\mathbb{V}^{*}, \mathbb{V}} & & \forall v_{m} \in \mathbb{V}_{m} \\
\left\langle B^{*} r_{m}, w_{n}\right\rangle_{\mathbb{U}^{*}, \mathbb{U}} & =0 & & \forall w_{n} \in \mathbb{U}_{n}
\end{array}\right.
$$

where the auxiliary variable $r_{m}$ is a discrete residual representer. Because of the monotone nonlinearity $J_{\mathbb{V}}$, we refer to (1.8) as a monotone mixed method. ${ }^{7}$

- (Theorem 4.5) Under the Fortin condition, the inexact MinRES method (1.5) (or equivalently (1.7) or (1.8)) has a unique solution that depends continuously on the data.

- (Theorem 4.14) Under the Fortin condition, the inexact MinRES method (1.5) is quasi-optimal, i.e., it satisfies the a priori error estimate:

$$
\left\|u-u_{n}\right\|_{\mathbb{U}} \leq C \inf _{w_{n} \in \mathbb{U}_{n}}\left\|u-w_{n}\right\|_{\mathbb{U}} .
$$

\footnotetext{
${ }^{4} \mathrm{~A}$ normed space $\mathbb{Y}$ is said to be strictly convex if, for all $y_{1}, y_{2} \in \mathbb{Y}$ such that $y_{1} \neq y_{2}$ and $\left\|y_{1}\right\|=\left\|y_{2}\right\|=1$, it holds that $\left\|\theta y_{1}+(1-\theta) y_{2}\right\|_{\mathbb{Y}}<1$ for all $\theta \in(0,1)$, see e.g., $[17,7,13]$.

${ }^{5}$ To give a specific example, if $\mathbb{V}=W_{0}^{1, p}(\Omega)$, then $J_{\mathbb{V}}$ is a (normalized) $p$-Laplace-type operator. We refer to Section 2 for details and other relevant properties.

${ }^{6}$ Natural injections $I_{m}: \mathbb{V}_{m} \rightarrow \mathbb{V}$ have been ommitted for simplicity; see Section 4.1.

${ }^{7}$ As might be expected, replacing $\mathbb{V}_{m}$ by $\mathbb{V}$ in (1.7), (1.8) gives equivalences to the ideal case (1.3).
} 
A major part of our analysis concerns the sharpening of the constant $C$ in (1.9). Indeed, a straightforward preliminary result (Corollary 4.8) is not sharp as it does not reduce to the known result $C=C_{\Pi} M_{B} / \gamma_{B}$, when restricting to Hilbert-space settings [26, Theorem 2.1], with $C_{\Pi}$ being a boundedness constant in Fortin's condition. To resolve the discrepancy, we improve the constant by including the dependence on the geometry of the involved Banach spaces. The proof of this sharper estimate is nontrivial, as it requires a suitable extension of a Hilbert-space technique due to $\mathrm{Xu}$ and Zikatanov [44] involving the classical identity $\|I-P\|=\|P\|$ for Hilbert-space projectors $P$, which is generally attributed to Kato [32] (cf. [42]). A key idea is the recent extension $\|I-P\| \leq C_{\mathrm{S}}\|P\|$ for Banach-space projectors by Stern [41], where $C_{\mathrm{S}}$ depends on the Banach-Mazur distance, however, since that extension applies to linear projectors, we generalize Stern's result to a suitable class of nonlinear projectors (see Lemma 3.3). As a by-product, we prove two novel a priori bounds for abstract best approximations and exact residual minimizers, which are of independent interest (see Propositions 3.5 and 3.17, and Corollaries 3.6 and 3.18, respectively).

1.3. Discussion: Unifying aspects and PDE implications. Let us emphasize that the above quasi-optimality theory generalizes and unifies Babuška's theory for Petrov-Galerkin methods [1], Guermond's theory for exact residual minimization [27], and the Hilbert-space theory for inexact residual minimization (including the DPG method) [26, 16, 3, 21]. For a schematic hierarchy with these connections, we refer to Remark 2 and Figure 2.

While the discretization theory developed in this work is abstract and applies to any well-posed operator equation, we mention some of its implications in the context of PDEs on bounded Lipschitz domains $\Omega \subset \mathbb{R}^{d}$. Firstly, the general Banach-space setting implies that one can directly consider PDEs in (non-standard) non-Hilbert settings. For example, it provides an immediate discretization theory for second-order elliptic operators $B: W_{0}^{1, p}(\Omega) \rightarrow W_{0}^{1, q}(\Omega)^{*}$ with $p>1$, such as the Laplacian or diffusion-convection-reaction operator; see [30, 28] for studies of their well-posedness, and [29] for a recent application of the inexact MinRes method. One can also utilize inexact residual minimization to directly approximate rough right-hand sides, essentially thinking of the operator $B$ being the identity in $\mathbb{V}^{*}=W^{1, q}(\Omega)^{*}$; see [35].

Secondly, one can consider first-order PDEs in a weak setting with $B: L^{p}(\Omega) \rightarrow$ $W_{B}^{q}(\Omega)^{*}$, where $W_{B}^{q}(\Omega)$ is a suitable graph space for $B$ (based on $L^{q}$ ). This setting has a solution space $\mathbb{U}=L^{p}(\Omega)$ that has very low regularity and accommodates discontinuous solutions (as typically expected for first-order PDEs). The recent work [36] explores this application in the context of the advection-reaction equation (or linear transport) with the additional benefit that the notorious Gibbs phenomena can be eliminated when $p \rightarrow 1^{+}$(cf. [29, 33]). We anticipate that the above-mentioned benefits may extend to other classes of linear PDEs, integro-partial differential equations and nonlocal PDEs, as well as to other Banach spaces. ${ }^{8}$

1.4. Outline of paper. The remainder of the paper is organized as follows.

- Section 2 is devoted to brief preliminaries on the duality mapping.

- Section 3 considers geometric constants in Banach spaces and sharpened a priori bounds involving these constants.

- Section 4 contains the complete analysis of the inexact MinREs method.

- Finally, the Appendix A contains some of the proofs in this work that were deemed too long to be included in the main body of the text.

${ }^{8}$ Cf. $[11,9,8]$ for nonlinear PDEs examples in Hilbert-space settings using a DPG approach. 
2. Preliminaries: Duality mappings. In this section we briefly review some relevant theory in the classical subject of duality mappings, which are required to obtain equivalent characterizations of (inexact) residual minimizers and best approximations. An extensive treatment on duality mappings can be found in Cioranescu [14]. ${ }^{9}$

Let $\mathbb{Y}$ be a normed vector space.

DEFinition 2.1 (Duality mapping).

(i) The multivalued mapping $\mathcal{J}_{\mathbb{Y}}: \mathbb{Y} \rightarrow 2^{\mathbb{Y}^{*}}$ defined by

$$
\mathcal{J}_{\mathbb{Y}}(y):=\left\{y^{*} \in \mathbb{Y}^{*}:\left\langle y^{*}, y\right\rangle_{\mathbb{Y}^{*}, \mathbb{Y}}=\|y\|_{\mathbb{Y}}^{2}=\left\|y^{*}\right\|_{\mathbb{Y}^{*}}^{2}\right\},
$$

is the duality mapping on $\mathbb{Y}$.

(ii) When $\mathcal{J}_{\mathbb{Y}}$ is a single-valued map, we use the notation $J_{\mathbb{Y}}: \mathbb{Y} \rightarrow \mathbb{Y}^{*}$ and call it the duality map on $\mathbb{Y}$, in other words, in that case

$$
\mathcal{J}_{\mathbb{Y}}(y)=\left\{J_{\mathbb{Y}}(y)\right\} \quad \text { such that } \quad\left\langle J_{\mathbb{Y}}(y), y\right\rangle_{\mathbb{Y}^{*}, \mathbb{Y}}=\|y\|_{\mathbb{Y}}^{2}=\left\|J_{\mathbb{Y}}(y)\right\|_{\mathbb{Y}^{*}}^{2} .
$$

Some classical properties of $\mathcal{J}_{\mathbb{Y}}$ (and $J_{\mathbb{Y}}$ ) are summarized in the following.

- $\mathcal{J}_{\mathbb{Y}}(y) \subset \mathbb{Y}^{*}$ is non-empty for all $y \in \mathbb{Y}$, and $\mathcal{J}_{\mathbb{Y}}(\cdot)$ is a homogeneous map.

- $\mathcal{J}_{\mathbb{Y}}(\cdot)$ is a single-valued map if and only if $\mathbb{Y}^{*}$ is strictly convex.

- $\mathcal{J}_{\mathbb{Y}}: \mathbb{Y} \rightarrow 2^{\mathbb{Y}^{*}}$ is surjective ${ }^{10}$ if and only if $\mathbb{Y}$ is reflexive.

- $\mathcal{J}_{\mathbb{Y}}$ is strictly monotone (hence injective) if and only if $\mathbb{Y}$ is strictly convex. Strict monotonicity is meant as follows: For all $y, z \in \mathbb{Y}, y \neq z$,

$$
\left\langle y^{*}-z^{*}, y-z\right\rangle_{\mathbb{Y}^{*}, \mathbb{Y}}>0 \quad \text { for any } y^{*} \in \mathcal{J}_{\mathbb{Y}}(y) \text { and } z^{*} \in \mathcal{J}_{\mathbb{Y}}(z) \text {. }
$$

Accordingly, when $\mathbb{Y}$ and $\mathbb{Y}^{*}$ are strictly convex and reflexive Banach spaces, referred to as the reflexive smooth setting, two important straightforward consequences are:

- $J_{\mathbb{Y}}: \mathbb{Y} \rightarrow \mathbb{Y}^{*}$ and $J_{\mathbb{Y}^{*}}: \mathbb{Y}^{*} \rightarrow \mathbb{Y}^{* *}$ are bijective.

- $J_{\mathbb{Y}^{*}}=\mathcal{I}_{\mathbb{Y}} \circ J_{\mathbb{Y}}^{-1}$, where $\mathcal{I}_{\mathbb{Y}}: \mathbb{Y} \rightarrow \mathbb{Y}^{* *}$ is the canonical injection. Briefly, $J_{\mathbb{Y}^{*}}=J_{\mathbb{Y}}^{-1}$, by means of canonical identification.

We also recall the following key characteristics of duality mappings:

- For any $y^{*} \in \mathcal{J}_{\mathbb{Y}}(y)$ (or $y^{*}=J_{\mathbb{Y}}(y)$ if single-valued), its norm supremum is achieved by $y$ itself, i.e.,

$$
\sup _{z \in \mathbb{Y} \backslash\{0\}} \frac{\left\langle y^{*}, z\right\rangle_{\mathbb{Y}^{*}, \mathbb{Y}}}{\|z\|_{\mathbb{Y}}}=\frac{\left\langle y^{*}, y\right\rangle_{\mathbb{Y}^{*}, \mathbb{Y}}}{\|y\|_{\mathbb{Y}}} \quad\left(=\|y\|_{\mathbb{Y}}\right) .
$$

- The duality mapping coincides with the subdifferential of $f_{\mathbb{Y}}: \mathbb{Y} \rightarrow \mathbb{R}$ defined by $f_{\mathbb{Y}}(\cdot):=\frac{1}{2}\|\cdot\|_{\mathbb{Y}}^{2}$, in other words, $\mathcal{J}_{\mathbb{Y}}(y)=\partial f_{\mathbb{Y}}(y)$, for all $y \in \mathbb{Y}$. Moreover, if $\mathbb{Y}^{*}$ is strictly convex, $f_{\mathbb{Y}}$ is Gâteaux differentiable with gradient $\nabla f_{\mathbb{Y}}(\cdot)$, hence, $J_{\mathbb{Y}}(y)=\nabla f_{\mathbb{Y}}(y)$.

Example 2.2 (The $L^{p}$ case). We recall here an explicit formula for the duality map in $L^{p}(\Omega)$, where $\Omega \subset \mathbb{R}^{d}, d \geq 1$. For $p \in(1,+\infty)$, the space $L^{p}(\Omega)$ is reflexive and strictly convex; see e.g. [14, Chapter II] and [7, Section 4.3]. For $v \in L^{p}(\Omega)$ the duality map is defined by the action:

$$
\left\langle J_{L^{p}(\Omega)}(v), w\right\rangle_{L^{q}(\Omega), L^{p}(\Omega)}:=\|v\|_{L^{p}(\Omega)}^{2-p} \int_{\Omega}|v|^{p-1} \operatorname{sign}(v) w, \quad \forall w \in L^{p}(\Omega),
$$

\footnotetext{
${ }^{9}$ Other relevant treatments in the context of nonlinear functional analysis are by Brezis [7, Chapter 1], Deimling [17, Section 12], Chidume [12, Chapter 3] and Zeidler [45, Chapter 32.3d], while an early treatment on duality mappings is by Lions [34, Chapter 2, Section 2.2].

${ }^{10}$ Surjective in the following sense: Every $y^{*} \in \mathbb{Y}^{*}$ belongs to a set $\mathcal{J}_{\mathbb{Y}}(y)$, for some $y \in \mathbb{Y}$.
} 


\footnotetext{
${ }^{11}$ In the case $p=1$, the formula in the right-hand side of (2.4) also works and defines an element in the set $\mathcal{J}_{L^{1}(\Omega)}(v)$. Note however that $L^{1}$ is not a special Banach space as discussed above.

${ }^{12}$ In fact, the Hahn-Banach extension is unique on account of strict convexity of $\mathbb{Y}^{*}$.
} 
Since the definition only makes sense when $\operatorname{dim} \mathbb{Y} \geq 2$, henceforth, whenever $C_{\mathrm{BM}}(\cdot)$ is written, we assume this to be the case. (Note that $\operatorname{dim} \mathbb{Y}=1$ is often an uninteresting trivial situation.)

Remark 3.2 (Elementary properties of $C_{\mathrm{BM}}$ ). It is known that $1 \leq C_{\mathrm{BM}}(\mathbb{Y}) \leq 2$, $C_{\mathrm{BM}}(\mathbb{Y})=1$ if and only if $\mathbb{Y}$ is a Hilbert space, and $C_{\mathrm{BM}}(\mathbb{Y})=2$ if $\mathbb{Y}$ is non-reflexive; see [41, Section 3]. For $\mathbb{Y}=\ell_{p}\left(\mathbb{R}^{2}\right), C_{\mathrm{BM}}(\mathbb{Y})=2^{\left|\frac{2}{p}-1\right|}$; cf. [43, Section II.E.8] and [31, Section 8], which is also true for $L^{p}$ and Sobolev spaces $W^{k, p}(k \in \mathbb{N})$; see [41].

The Banach-Mazur constant is used in the Lemma below to state a fundamental estimate for an abstract nonlinear projector. This nonlinear projector estimate, is an extension of Kato's identity $\|I-P\|=\|P\|$ for Hilbert-space projectors [32], and a generalization of the estimate in [41, Theorem 3] (for linear Banach-space projectors).

LEMma 3.3 (Nonlinear projector estimate). Let $\mathbb{Y}$ be a normed space, $I: \mathbb{Y} \rightarrow \mathbb{Y}$ the identity and $Q: \mathbb{Y} \rightarrow \mathbb{Y}$ a nonlinear operator such that:

(i) $Q$ is a nontrivial projector: $0 \neq Q=Q \circ Q \neq I$.

(ii) $Q$ is homogeneous: $Q(\lambda y)=\lambda Q(y), \quad \forall y \in \mathbb{Y}$ and $\forall \lambda \in \mathbb{R}$.

(iii) $Q$ is bounded in the sense that $\|Q\|:=\sup _{y \in \mathbb{Y} \backslash\{0\}} \frac{\|Q(y)\|_{\mathbb{Y}}}{\|y\|_{\mathbb{Y}}}<+\infty$.

(iv) $Q$ is a quasi-linear projector in the sense that

$$
Q(y)=Q(Q(y)+\eta(I-Q)(y)), \quad \text { for any } \eta \in \mathbb{R} \text { and any } y \in \mathbb{Y} .
$$

Then the nonlinear operator $I-Q$ is also bounded and satisfies

$$
\|I-Q\| \leq C_{\mathrm{S}}\|Q\|, \quad \text { with } C_{\mathrm{S}}:=\min \left\{1+\|Q\|^{-1}, C_{\mathrm{BM}}(\mathbb{Y})\right\} .
$$

Proof. The proof of this result follows closely Stern [41, Proof of Theorem 3]. Although Stern considers linear projectors, his result generalizes to projectors with the properties in (i)-(iv). See Section A.1 for the complete proof.

Remark 3.4 (Quasi-linear projectors). Requirement (iv) in Lemma 3.3 is a key nonlinear property. We point out that it is satisfied by linear projectors, by bestapproximation projectors, by $I$ minus best-approximation projectors (as in the proof of Proposition 3.5), and by (inexact) nonlinear PG projectors (see Corollary 4.13).

3.2. First a priori bounds for best approximations and residual minimizers. By applying Lemma 3.3, we now obtain a priori bounds for best approximations and exact residual minimizers.

Proposition 3.5 (Best approximation: A priori bound I). Let $\mathbb{Y}$ be a Banach space and $\mathbb{M} \subset \mathbb{Y}$ a closed subspace. Suppose $y_{0} \in \mathbb{M}$ is a best approximation in $\mathbb{M}$ of a given $y \in \mathbb{Y}$, i.e.,

$$
\left\|y-y_{0}\right\|_{\mathbb{Y}} \leq\left\|y-z_{0}\right\|_{\mathbb{Y}}, \quad \forall z_{0} \in \mathbb{M},
$$

then $y_{0}$ satisfies the a priori bound:

$$
\left\|y_{0}\right\|_{\mathbb{Y}} \leq C_{\mathrm{BM}}(\mathbb{Y})\|y\|_{\mathbb{Y}} .
$$

Proof. We assume $\mathbb{M} \neq\{0\}$ and $\mathbb{M} \neq \mathbb{Y}$ (otherwise the result is trivial). Consider a (nonlinear) map $P^{\perp}: \mathbb{Y} \rightarrow \mathbb{Y}$ such that $P^{\perp}(y)=y-y_{0}$, where $y_{0} \in \mathbb{M}$ is a best 
approximation to $y \in \mathbb{Y}$. The map $P^{\perp}$ can be chosen in a homogeneous way, i.e., satisfying $\lambda P^{\perp}(y)=P^{\perp}(\lambda y)$ for any $\lambda \in \mathbb{R}$. Observe that

$$
\left\|P^{\perp}(y)\right\|_{\mathbb{Y}}=\left\|y-y_{0}\right\|_{\mathbb{Y}} \leq\|y-0\|_{\mathbb{Y}}=\|y\|_{\mathbb{Y}} .
$$

Hence, $\left\|P^{\perp}\right\| \leq 1$. Additionally, it can be verified that $P^{\perp}\left(P^{\perp}(y)\right)=y-y_{0}-0=$ $P^{\perp}(y)$. Thus, $Q=P^{\perp}$ satisfies (i)-(iii) of Lemma 3.3. To verify requirement (iv), notice that for any $\eta \in \mathbb{R}$,

$$
P^{\perp}\left(P^{\perp}(y)+\eta\left(I-P^{\perp}\right)(y)\right)=P^{\perp}\left(y-y_{0}+\eta y_{0}\right)=y-y_{0},
$$

since $\eta y_{0}$ is a best approximation in $\mathbb{M}$ to $y-y_{0}+\eta y_{0}$. Therefore, by Lemma 3.3,

$$
\left\|y_{0}\right\|_{\mathbb{Y}}=\left\|\left(I-P^{\perp}\right) y\right\|_{\mathbb{Y}} \leq \min \left\{1+\left\|P^{\perp}\right\|^{-1}, C_{\mathrm{BM}}(\mathbb{Y})\right\}\left\|P^{\perp}\right\|\|y\|_{\mathbb{Y}},
$$

and (3.1) follows since $\left\|P^{\perp}\right\| \leq 1$ and $C_{\mathrm{BM}}(\mathbb{Y}) \leq 2$.

Corollary 3.6 (Residual minimization: A priori bound I). Let $u_{n} \in \mathbb{U}_{n}$ be a solution of the exact MINRES problem (1.3), then $u_{n}$ satisfies the a priori bound:

$$
\left\|u_{n}\right\|_{\mathbb{U}} \leq \frac{C_{\mathrm{BM}}\left(\mathbb{V}^{*}\right)}{\gamma_{B}}\|f\|_{\mathbb{V}^{*}} .
$$

Proof. First note that $u_{n}$ is a best approximation to $u$ in the (energy) norm $\|\cdot\|_{\mathbb{E}}:=\|B(\cdot)\|_{\mathbb{V}^{*}}\left(\right.$ which is an equivalent norm to $\|\cdot\|_{\mathbb{U}}$ because of $\left.(1.2)\right)$, indeed

$$
\left\|u-u_{n}\right\|_{\mathbb{E}}=\left\|B u-B u_{n}\right\|_{\mathbb{V}^{*}}=\left\|f-B u_{n}\right\|_{\mathbb{V}^{*}} \leq\left\|f-B w_{n}\right\|_{\mathbb{V}^{*}}=\left\|u-w_{n}\right\|_{\mathbb{E}},
$$

for any $w_{n} \in \mathbb{U}_{n}$. Thus, applying Proposition 3.5 shows that

$$
\left\|u_{n}\right\|_{\mathbb{U}} \leq \frac{1}{\gamma_{B}}\left\|u_{n}\right\|_{\mathbb{E}} \leq \frac{C_{\mathrm{BM}}\left(\mathbb{V}^{*}\right)}{\gamma_{B}}\|u\|_{\mathbb{E}}=\frac{C_{\mathrm{BM}}\left(\mathbb{V}^{*}\right)}{\gamma_{B}}\|f\|_{\mathbb{V}^{*}} .
$$

Remark 3.7 (Sharpness of (3.1)). The bound in (3.1) improves the classical bound $\left\|y_{0}\right\|_{\mathbb{Y}} \leq 2\|y\|_{\mathbb{Y}}$ (see e.g., [40, Sec. 10.2]), in the sense that (3.1) shows an explicit dependence on the geometry of the underlying Banach space. In particular, (3.1) contains the standard result $\left\|y_{0}\right\|_{\mathbb{Y}} \leq\|y\|_{\mathbb{Y}}$ for a Hilbert space, as well as the classical bound $\left\|y_{0}\right\|_{\mathbb{Y}} \leq 2\|y\|_{\mathbb{Y}}$ for non-reflexive spaces such as $\ell_{1}\left(\mathbb{R}^{2}\right)$ and $\ell_{\infty}\left(\mathbb{R}^{2}\right)$ (for which the bound is indeed sharp; see Example 3.8). However, (3.1) need not be sharp for intermediate spaces; see Example 3.11.

Example $3.8\left(\ell^{1}\left(\mathbb{R}^{2}\right)\right)$. In $\mathbb{R}^{2}$ with the norm $\left\|\left(x_{1}, x_{2}\right)\right\|_{1}=\left|x_{1}\right|+\left|x_{2}\right|$, i.e. $\mathbb{Y}=$ $\ell^{1}\left(\mathbb{R}^{2}\right)$, the best approximation of the point $(1,0)$ over the line $\{(t, t): t \in \mathbb{R}\}$ is the whole segment $\{(t, t): t \in[0,1]\}$. Moreover, the point $(1,1)$ is a best approximation and $\|(1,1)\|_{1}=2=2\|(0,1)\|_{1}$. Since the Banach-Mazur constant equals 2, Eq. (3.1) is sharp for this example.

3.3. Asymmetric-orthogonality constant and strengthened triangle inequality. We now introduce a new geometric constant, which will appear in alternative a priori bounds for best approximations and (inexact) residual minimizers. 
DEFINITION 3.9 (Asymmetric-orthogonality constant). Let $\mathbb{Y}$ be a normed vector space with $\operatorname{dim} \mathbb{Y} \geq 2$. The asymmetric-orthogonality constant is defined by: ${ }^{13}$

$$
C_{\mathrm{AO}}(\mathbb{Y}):=\sup _{\substack{\left(z_{0}, z\right) \in \mathcal{O}_{\mathbb{Y}} \\ z_{0}^{*} \in \mathcal{J}_{\mathbb{Y}}\left(z_{0}\right)}} \frac{\left\langle z_{0}^{*}, z\right\rangle_{\mathbb{Y}^{*}, \mathbb{Y}}}{\|z\|_{\mathbb{Y}}\left\|z_{0}\right\|_{\mathbb{Y}}},
$$

where the above supremum is taken over the set $\mathcal{O}_{\mathbb{Y}}$ consisting of all pairs $\left(z_{0}, z\right)$ which are orthogonal in the following sense:

$$
\mathcal{O}_{\mathbb{Y}}:=\left\{\left(z_{0}, z\right) \in \mathbb{Y} \times \mathbb{Y}: \exists z^{*} \in \mathcal{J}_{\mathbb{Y}}(z) \text { satisfying }\left\langle z^{*}, z_{0}\right\rangle_{\mathbb{Y}^{*}, \mathbb{Y}}=0\right\} .
$$

Remark 3.10 (Elementary properties of $C_{\mathrm{AO}}$ ). The constant $C_{\mathrm{AO}}(\mathbb{Y})$ is a geometric constant since it measures the degree to which the orthogonality relation (3.6) fails to be symmetric. It is easy to see that $0 \leq C_{\mathrm{AO}}(\mathbb{Y}) \leq 1$. If $\mathbb{Y}$ is a Hilbert space, then $C_{\mathrm{AO}}(\mathbb{Y})=0$, since then $J_{\mathbb{Y}}(\cdot)$ coincides with the self-adjoint Riesz map, and $\left\langle J_{\mathbb{Y}}(\cdot), \cdot\right\rangle_{\mathbb{Y} * \mathbb{Y}}$ coincides with the (symmetric) inner product in $\mathbb{Y}$. On the other hand, the maximal value $C_{\mathrm{AO}}(\mathbb{Y})=1$ holds for example for $\mathbb{Y}=\ell_{1}\left(\mathbb{R}^{2}\right)$. Indeed taking $z_{0}=(1,-1)$ and $z=(\alpha, 1)$, with $\alpha>0$, then $(2,-2) \in \mathcal{J}_{\mathbb{Y}}\left(z_{0}\right)$ and $(1+\alpha, 1+\alpha) \in$ $\mathcal{J}_{\mathbb{Y}}(z)$, so that upon taking $\alpha \rightarrow+\infty$ one obtains $\left\langle z_{0}^{*}, z\right\rangle_{\mathbb{Y}^{*}, \mathbb{Y}} /\left(\left\|z_{0}\right\|_{\mathbb{Y}}\|z\|_{\mathbb{Y}}\right) \rightarrow 1$.

Example $3.11\left(C_{\mathrm{AO}}\left(\ell_{p}\right)\right)$. Consider the Banach space $\ell_{p} \equiv \ell_{p}\left(\mathbb{R}^{2}\right)$ with $1<p<$ $+\infty$ (i.e., $\mathbb{R}^{2}$ endowed with the $p$-norm). In this case the duality map is given by:

$$
\left\langle J_{\ell^{p}}\left(x_{1}, x_{2}\right),\left(y_{1}, y_{2}\right)\right\rangle_{\left(\ell_{p}\right)^{*}, \ell_{p}}=\left\|\left(x_{1}, x_{2}\right)\right\|_{\ell_{p}}^{2-p} \sum_{i=1}^{2}\left|x_{i}\right|^{p-1} \operatorname{sign}\left(x_{i}\right) y_{i},
$$

for all $\left(x_{1}, x_{2}\right),\left(y_{1}, y_{2}\right) \in \mathbb{R}^{2}$, which allows the computation of $C_{\mathrm{AO}}\left(\ell_{p}\right)$ as a constrained maximization problem. Figure 1 shows the dependence of $C_{\mathrm{AO}}\left(\ell_{p}\right)$ versus $p-1$. It also illustrates the Banach-Mazur constant $C_{\mathrm{BM}}\left(\ell_{p}\right)$ and the best-approximation projection constant $C_{\text {best }}\left(\ell_{p}\right):=\max _{u \in \ell_{p}\left(\mathbb{R}^{2}\right)}\left\|u_{n}\right\| /\|u\|$, with $u_{n}$ the best approximation to $u$ on the worst 1 -dimensional subspace of $\ell_{p}\left(\mathbb{R}^{2}\right)$. The figure shows that

$$
C_{\text {best }}\left(\ell_{p}\right)<C_{\mathrm{BM}}\left(\ell_{p}\right)<1+C_{\mathrm{AO}}\left(\ell_{p}\right)
$$

except for $p=1,2$ and $+\infty$, for which they coincide. ${ }^{14}$

We conclude our discussion of $C_{\mathrm{AO}}$ with a Lemma describing three important properties that are going to be used later in Section 4.4.

Lemma $3.12\left(C_{\mathrm{AO}}\right.$ in reflexive smooth setting). Assume $\mathbb{Y}$ and $\mathbb{Y}^{*}$ are strictly convex and reflexive Banach spaces. The following properties hold true:

(i) $C_{\mathrm{AO}}(\mathbb{Y})=\sup _{\left(z_{0}, z\right) \in \mathbb{Y} \times \mathbb{Y}:\left\langle J_{\mathbb{Y}}(z), z_{0}\right\rangle_{\mathbb{Y}^{*}, \mathbb{Y}}=0} \frac{\left\langle J_{\mathbb{Y}}\left(z_{0}\right), z\right\rangle_{\mathbb{Y}^{*}, \mathbb{Y}}}{\|z\|_{\mathbb{Y}}\left\|z_{0}\right\|_{\mathbb{Y}}}$.

(ii) $C_{\mathrm{AO}}\left(\mathbb{Y}^{*}\right)=C_{\mathrm{AO}}(\mathbb{Y})$.

(iii) $C_{\mathrm{AO}}(\mathbb{M})=C_{\mathrm{AO}}(\mathbb{Y})$, for any closed subspace $\mathbb{M} \subset \mathbb{Y}$ endowed with norm $\|\cdot\|_{\mathbb{Y}}$.

Proof. See Section A.2.

\footnotetext{
${ }^{13} \mathrm{As}$ in the case of $C_{\mathrm{BM}}(\mathbb{Y}), C_{\mathrm{AO}}(\mathbb{Y})$ only makes sense when $\operatorname{dim} \mathbb{Y} \geq 2$. Therefore as before, whenever $C_{\mathrm{AO}}(\cdot)$ is written, we assume this to be the case.

${ }^{14}$ It is unknown if (3.7) holds more generally than in this example.
} 


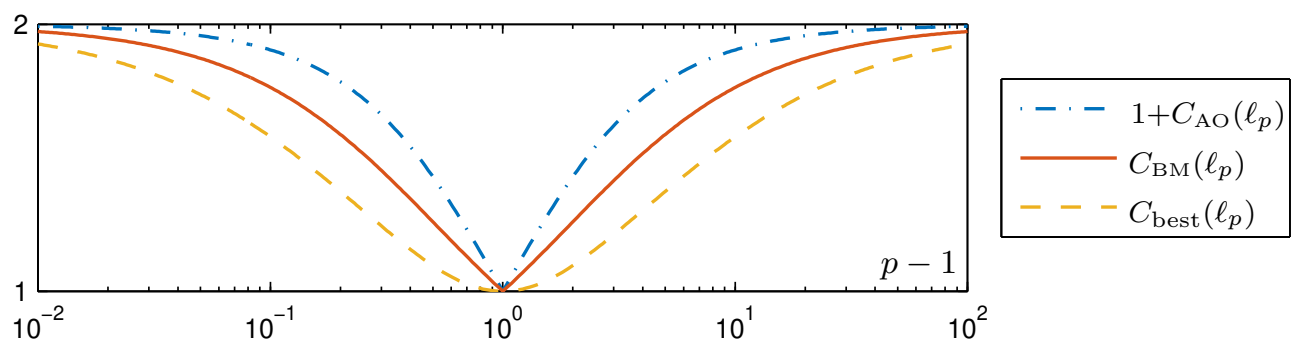

FIG. 1. Three different geometric constants and its dependence on $p-1$.

Example $3.13\left(C_{\mathrm{AO}}\left(L^{p}\right)\right)$. Let $\Omega \subset \mathbb{R}^{d}$ be an open set and consider the Banach space $\mathbb{Y}:=L^{p}(\Omega), 1<p<+\infty$. Let $\Omega_{1}$ and $\Omega_{2}$ be two open bounded disjoint subsets. Define the functions $f_{i} \in L^{p}(\Omega)(i=1,2)$ by $f_{i}:=\left|\Omega_{i}\right|^{-\frac{1}{p}} \mathbb{1}_{\Omega_{i}}$ and let $\mathbb{M}:=\operatorname{span}\left\{f_{1}, f_{2}\right\} \subset \mathbb{Y}$. It is easy to see that $\mathbb{M}$ is isometrically isomorphic to $\ell_{p}\left(\mathbb{R}^{2}\right)$ and thus, using Lemma 3.12(iii), we have $C_{\mathrm{AO}}\left(\ell_{p}\right)=C_{\mathrm{AO}}(\mathbb{M})=C_{\mathrm{AO}}\left(L^{p}\right)$.

We now use the constant $C_{\mathrm{AO}}$ to state a strengthened triangle inequality. ${ }^{15}$ This inequality can be thought of as an extension of the inequality $\left\|y_{0}\right\| \leq\|y\|$ in Hilbert spaces whenever $\left(y-y_{0}, y_{0}\right)=0$. In the worst Banach spaces (having $C_{\mathrm{AO}}=1$ ), the below inequality reduces to the standard triangle inequality $\left\|y_{0}\right\| \leq\|y\|+\left\|y-y_{0}\right\|$.

Lemma 3.14 (Strengthened triangle inequality). Let $\mathbb{Y}$ be a Banach space. Suppose $y_{0}, y \in \mathbb{Y}$ such that

$$
\exists z^{*} \in \mathcal{J}_{\mathbb{Y}}\left(y-y_{0}\right) \text { satisfying }\left\langle z^{*}, y_{0}\right\rangle_{\mathbb{Y}^{*}, \mathbb{Y}}=0
$$

(or simply $\left\langle J_{\mathbb{Y}}\left(y-y_{0}\right), y_{0}\right\rangle_{\mathbb{Y}^{*}, \mathbb{Y}}=0$ in the case $\mathbb{Y}^{*}$ is strictly convex), then

$$
\left\|y_{0}\right\|_{\mathbb{Y}} \leq\|y\|_{\mathbb{Y}}+C_{\mathrm{AO}}(\mathbb{Y})\left\|y-y_{0}\right\|_{\mathbb{Y}} \cdot
$$

Proof. If $y_{0}=0$ or $y_{0}=y$, the result is obvious. Note that $y=0$ implies $y_{0}=0$, hence is also a trivial situation. Thus, assume $0 \neq y_{0} \neq y \neq 0$. Consider any $y_{0}^{*} \in \mathcal{J}_{\mathbb{Y}}\left(y_{0}\right)\left(\right.$ or $y_{0}^{*}=J_{\mathbb{Y}}\left(y_{0}\right)$ when $\mathbb{Y}^{*}$ is strictly convex), then

$$
\left\|y_{0}\right\|_{\mathbb{Y}}=\frac{\left\langle y_{0}^{*}, y_{0}\right\rangle_{\mathbb{Y}^{*}, \mathbb{Y}}}{\left\|y_{0}\right\|_{\mathbb{Y}}}=\frac{\left\langle y_{0}^{*}, y\right\rangle_{\mathbb{Y}^{*}, \mathbb{Y}}}{\left\|y_{0}\right\|_{\mathbb{Y}}}-\frac{\left\langle y_{0}^{*}, y-y_{0}\right\rangle_{\mathbb{Y}^{*}, \mathbb{Y}}}{\left\|y_{0}\right\|}\left\|y-y_{\mathbb{Y}}\right\| y-y_{0} \|_{\mathbb{Y}}
$$

Because $\left\langle z^{*}, y_{0}\right\rangle_{\mathbb{Y}^{*}, \mathbb{Y}}=0$ by assumption, the absolute value of the second fraction on the right-hand side is bounded by $C_{\mathrm{AO}}(\mathbb{Y})$, from which the proof follows.

Example $3.15\left(\ell^{1}\left(\mathbb{R}^{2}\right)\right.$ continued). Recall from Example 3.8, the points $y_{0}=$ $(1,1)$ and $y=(1,0)$ in $\ell^{1}\left(\mathbb{R}^{2}\right)$, and observe that $\left\|y_{0}\right\|_{1}=\|y\|_{1}+\left\|y-y_{0}\right\|_{1}$. Define $z^{*}=$ $(1,-1)$ and note that $z^{*} \in \mathcal{J}_{\ell^{1}}\left(y-y_{0}\right) \in \ell^{\infty}\left(\mathbb{R}^{2}\right)$ and $\left\langle z^{*}, y_{0}\right\rangle=0$. Hence, since $C_{\mathrm{AO}}\left(\ell_{1}\right)=1$, Eq. (3.8) is sharp in this case.

3.4. Second a priori bounds for best approximations and residual minimizers. The second set of a priori bounds for best approximations and exact residual minimizers involves the asymmetric-orthogonality constant, and is based on the following key characterization for best approximations.

\footnotetext{
${ }^{15}$ So named for its similarity to the strengthened Cauchy-Schwartz inequality; see, e.g. [22].
} 
Lemma 3.16 (Best approximation characterization). Let $\mathbb{Y}$ be a Banach space, and $y \in \mathbb{Y}$. Suppose $\mathbb{M} \subset \mathbb{Y}$ is a closed subspace, then the following are equivalent:

(i) $y_{0}$ is a best approximation in $\mathbb{M}$ to $y$, i.e., $y_{0}=\arg \min _{z_{0} \in \mathbb{M}}\left\|y-z_{0}\right\|_{\mathbb{Y}}$.

(ii) $\exists z^{*} \in \mathcal{J}_{\mathbb{Y}}\left(y-y_{0}\right)$ that annihilates $\mathbb{M}$, i.e., $\left\langle z^{*}, z_{0}\right\rangle_{\mathbb{Y}^{*}, \mathbb{Y}}=0, \forall z_{0} \in \mathbb{M}$.

Proof. In case of $y \in \mathbb{Y} \backslash \mathbb{M}$ see, e.g., Singer [39] or Braess [6]. The case of $y \in \mathbb{M}$ is trivial, because in that case $y_{0}=y$ and one can choose $z^{*}=0$.

Proposition 3.17 (Best approximation: A priori bound II). Suppose the conditions of Proposition 3.5. Then $y_{0}$ satisfies the a priori bound:

$$
\left\|y_{0}\right\|_{\mathbb{Y}} \leq\left(1+C_{\mathrm{AO}}(\mathbb{Y})\right)\|y\|_{\mathbb{Y}} .
$$

Proof. If $y_{0}=0$ or $y_{0}=y$, then the result is obvious. Hence, consider $\left\|y_{0}\right\|_{\mathbb{Y}}>0$ and $\left\|y-y_{0}\right\|_{\mathbb{Y}}>0$. Next, by Lemma 3.16, there exists $z^{*} \in \mathcal{J}_{\mathbb{Y}}\left(y-y_{0}\right)$ which annihilates $\mathbb{M}$, hence in particular $\left\langle z^{*}, y_{0}\right\rangle_{\mathbb{Y} *, \mathbb{Y}}=0$. Conclude by applying the strengthened triangle inequality (Lemma 3.14), and recalling that $\left\|y-y_{0}\right\|_{\mathbb{Y}} \leq\|y\|_{\mathbb{Y}}$ (see (3.2)).

Corollary 3.18 (Residual minimization: A priori bound II). Let $u_{n} \in \mathbb{U}_{n}$ be a solution of the exact MINRES problem (1.3), then $u_{n}$ satisfies the a priori bound:

$$
\left\|u_{n}\right\|_{\mathbb{U}} \leq \frac{\left(1+C_{\mathrm{AO}}(\mathbb{V})\right)}{\gamma_{B}}\|f\|_{\mathbb{V} *} .
$$

Proof. Similar to the proof of Corollary 3.6 (but now uses Proposition 3.17) and Lemma 3.12(ii).

4. Analysis of the inexact method. In this section, we present the analysis for the inexact MinREs method (1.5).

4.1. Equivalent formulations. We summarize the equivalent formulations in the following result, which utilizes the duality map (recall from Section 2).

TheOREM 4.1 (Equivalent characterizations). Let $\mathbb{U}$ and $\mathbb{V}$ be two Banach spaces and let $B: \mathbb{U} \rightarrow \mathbb{V}^{*}$ be a linear, continuous and bounded-below operator. Assume that $\mathbb{V}$ and $\mathbb{V}^{*}$ are reflexive and strictly convex. Consider finite-dimensional subspaces $\mathbb{U}_{n} \subset \mathbb{U}$ and $\mathbb{V}_{m} \subset \mathbb{V}$, together with the natural injections $I_{m}: \mathbb{V}_{m} \rightarrow \mathbb{V}$ and $I_{m}^{*}$ : $\mathbb{V}^{*} \rightarrow\left(\mathbb{V}_{m}\right)^{*}$, and duality maps $J_{\mathbb{V}}: \mathbb{V} \rightarrow \mathbb{V}^{*}$ and $J_{\mathbb{V}_{m}}: \mathbb{V}_{m} \rightarrow\left(\mathbb{V}_{m}\right)^{*}$. Given $f \in \mathbb{V}^{*}$, the following statements are equivalent: ${ }^{16}$

(i) $u_{n} \in \mathbb{U}_{n}$ minimizes the discrete residual, i.e.,

$$
\left\|I_{m}^{*}\left(f-B u_{n}\right)\right\|_{\left(\mathbb{V}_{m}\right)^{*}}=\min _{w_{n} \in \mathbb{U}_{n}}\left\|I_{m}^{*}\left(f-B w_{n}\right)\right\|_{\left(\mathbb{V}_{m}\right)^{*}},
$$

and $r_{m}=J_{\mathbb{V}_{m}}^{-1} \circ I_{m}^{*}\left(f-B u_{n}\right)$ is the associated minimal-residual representative.

(ii) $\left(r_{m}, u_{n}\right) \in \mathbb{V}_{m}^{m} \times \mathbb{U}_{n}$ solves the discrete mixed problem:

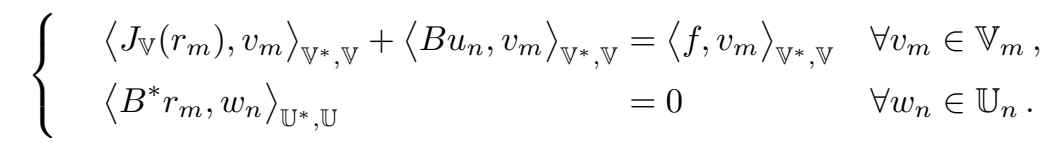

(iii) $u_{n} \in \mathbb{U}_{n}$ solves the inexact non-linear Petrov-Galerkin discretization:

$$
\left\langle\nu_{n}, I_{m} J_{\mathbb{V}_{m}}^{-1} \circ I_{m}^{*}\left(f-B u_{n}\right)\right\rangle_{\mathbb{\mathbb { V } ^ { * } , \mathbb { V }}}=0, \quad \forall \nu_{n} \in B \mathbb{U}_{n}
$$

$$
\text { and } r_{m}=J_{\mathbb{V}_{m}}^{-1} \circ I_{m}^{*}\left(f-B u_{n}\right) \text {. }
$$

\footnotetext{
${ }^{16}$ The presumed existence of solutions in these statements will be established in Theorem 4.5.
} 
(iv) $\left(r_{m}, u_{n}\right) \in \mathbb{V}_{m} \times \mathbb{U}_{n}$ solves the discrete saddle-point problem:

$$
\mathcal{L}\left(r_{m}, u_{n}\right)=\min _{v_{m} \in \mathbb{V}_{m}} \max _{w_{n} \in \mathbb{U}_{n}} \mathcal{L}\left(v_{m}, w_{n}\right),
$$

where the Lagrangian $\mathcal{L}: \mathbb{V} \times \mathbb{U} \rightarrow \mathbb{R}$ is defined by:

$$
\mathcal{L}(v, w):=\frac{1}{2}\|v\|_{\mathbb{V}}^{2}-\langle f, v\rangle_{\mathbb{V}^{*}, \mathbb{V}}+\left\langle B^{*} v, w\right\rangle_{\mathbb{U}^{*}, \mathbb{U}} \cdot
$$

Proof. Step (i) $\Rightarrow$ (ii). To verify (4.2a), notice the following direct equivalences:

$$
\begin{aligned}
& r_{m}=J_{\mathbb{V}_{m}}^{-1} \circ I_{m}^{*}\left(f-B u_{n}\right) \\
\Leftrightarrow & J_{\mathbb{V}_{m}}\left(r_{m}\right)=I_{m}^{*}\left(f-B u_{n}\right)
\end{aligned}
$$

(by Lemma 2.3) $\Leftrightarrow I_{m}^{*} J_{\mathbb{V}}\left(I_{m} r_{m}\right)=I_{m}^{*}\left(f-B u_{n}\right)$

$$
\Leftrightarrow\left\langle J_{\mathbb{V}}\left(r_{m}\right), v_{m}\right\rangle_{\mathbb{V}^{*}, \mathbb{V}}+\left\langle B u_{n}, v_{m}\right\rangle_{\mathbb{V}^{*}, \mathbb{V}}=\left\langle f, v_{m}\right\rangle_{\mathbb{V}^{*}, \mathbb{V}}, \forall v_{m} \in \mathbb{V}_{m} .
$$

Next, to verify $(4.2 \mathrm{~b})$, first recall the identification $J_{\left(\mathbb{V}_{m}\right)^{*}}=J_{\mathbb{V}_{m}}^{-1}$ due to the reflexive smooth setting. Now, if $u_{n} \in \mathbb{U}_{n}$ is a minimizer of (4.1) and $r_{m}=J_{\mathbb{V}_{m}}^{-1}$ 。 $I_{m}^{*}\left(f-B u_{n}\right)$, then by Lemma 3.16, with $\mathbb{M}=I_{m}^{*} B \mathbb{U}_{n} \subset\left(\mathbb{V}_{m}\right)^{*}=\mathbb{Y}, r_{m}$ satisfies:

$0=\left\langle I_{m}^{*} B w_{n}, r_{m}\right\rangle_{\left(\mathbb{V}_{m}\right)^{*}, \mathbb{V}_{m}}=\left\langle B w_{n}, I_{m} r_{m}\right\rangle_{\mathbb{V}^{*}, \mathbb{V}}=\left\langle B^{*} r_{m}, w_{n}\right\rangle_{\mathbb{U}^{*}, \mathbb{U}}, \quad \forall w_{n} \in \mathbb{U}_{n}$.

Step (ii) $\Rightarrow$ (iii). If $\left(u_{n}, r_{m}\right) \in \mathbb{U}_{n} \times \mathbb{V}_{m}$ is a solution of (4.2), then, by the direct equivalences in Step (i) $\Rightarrow$ (ii), $r_{m}=J_{\mathbb{V}_{m}}^{-1} \circ I_{m}^{*}\left(f-B u_{n}\right)$, and (4.2b) is nothing but (4.3).

Step (iii) $\Rightarrow$ (i). Observe that for any $w_{n} \in \mathbb{U}_{n}$ we have :

$$
\left\|I_{m}^{*}\left(f-B u_{n}\right)\right\|_{\left(\mathbb{V}_{m}\right)^{*}}=\sup _{v_{m} \in \mathbb{V}_{m}} \frac{\left\langle I_{m}^{*}\left(f-B u_{n}\right), v_{m}\right\rangle_{\left(\mathbb{V}_{m}\right)^{*}, \mathbb{V}_{m}}}{\left\|v_{m}\right\|_{\mathbb{V}}}
$$

$$
\begin{aligned}
& =\frac{\left\langle J_{\mathbb{V}_{m}}\left(r_{m}\right), r_{m}\right\rangle_{\left(\mathbb{V}_{m}\right)^{*}, \mathbb{V}_{m}}}{\left\|r_{m}\right\|_{\mathbb{V}}}=\frac{\left\langle I_{m}^{*}\left(f-B u_{n}\right), r_{m}\right\rangle_{\left(\mathbb{V}_{m}\right)^{*}, \mathbb{V}_{m}}}{\left\|r_{m}\right\|_{\mathbb{V}}} \\
& =\frac{\left\langle I_{m}^{*}\left(f-B w_{n}\right), r_{m}\right\rangle_{\left(\mathbb{V}_{m}\right)^{*}, \mathbb{V}_{m}}}{\left\|r_{m}\right\|_{\mathbb{V}}} \leq\left\|I_{m}^{*}\left(f-B w_{n}\right)\right\|_{\left(\mathbb{V}_{m}\right)^{*}} .
\end{aligned}
$$

Thus, $u_{n}$ is a minimizer of (4.1).

Step (ii) $\Leftrightarrow$ (iv). This is a classical result; see, e.g., Ekeland \& Témam [23, Chapter VI, Proposition 1.6] (use that $v \mapsto \frac{1}{2}\|v\|_{\mathbb{V}}^{2}$ is (strictly) convex, and that it is Gâteaux differentiable, owing to strict convexity of $\mathbb{V}^{*}$ ).

Remark $4.2\left(\mathbb{V}_{m}=\mathbb{V}\right)$. All the equivalences of Theorem 4.1 still hold true when $\mathbb{V}_{m}=\mathbb{V}$, which are relevant to the exact (or ideal) MinRes problem (1.3).

Remark 4.3 (Optimal test-space norm). As proposed in [46] (cf. [16]), if $\mathbb{V}$ is reflexive and $B$ is bijective (hence $B^{*}: \mathbb{V} \rightarrow \mathbb{U}^{*}$ is bijective), one can endow the space $\mathbb{V}$ with the equivalent optimal norm $\|\cdot\|_{\mathbb{V}_{\text {opt }}}:=\left\|B^{*}(\cdot)\right\|_{\mathbb{U}^{*}}$. In that case, the exact MinRES problem (1.3) precisely coincides with finding the best approximation in $\mathbb{U}_{n}$ to $u$ measured in $\|\cdot\|_{\mathbb{U}}$, i.e., $\left\|u-u_{n}\right\|_{\mathbb{U}}=\left\|f-B u_{n}\right\|_{\left(\mathbb{V}_{\mathrm{opt}}\right)^{*}}=\inf _{w_{n} \in \mathbb{U}_{n}}\left\|u-w_{n}\right\|_{\mathbb{U}}$. Besides, the duality map for this topology satisfies $J_{\mathbb{V}_{\mathrm{opt}}}(\cdot)=B J_{\mathbb{U}}^{-1} \circ B^{*}(\cdot)$. 
4.2. Well-posedness of the inexact method. We now focus on the monotone mixed method (1.8) (see also (4.2)), as this is the most convenient equivalent formulation for the ensuing well-posedness and error analysis.

Assumption 4.4 (Fortin condition). Let $\left\{\left(\mathbb{U}_{n}, \mathbb{V}_{m}\right)\right\}$ be a family of discrete subspace pairs, where $\mathbb{U}_{n} \subset \mathbb{U}$ and $\mathbb{V}_{m} \subset \mathbb{V}$. For each pair $\left(\mathbb{U}_{n}, \mathbb{V}_{m}\right)$ in this family, there exists an operator $\Pi_{n, m}: \mathbb{V} \rightarrow \mathbb{V}_{m}$ and constants $C_{\Pi}>0$ and $D_{\Pi}>0$ (independent of $n$ and $m$ ) such that the following conditions are satisfied:

$$
\begin{cases}\left\|\Pi_{n, m} v\right\|_{\mathbb{V}} \leq C_{\Pi}\|v\|_{\mathbb{V}}, & \forall v \in \mathbb{V}, \\ \left\|\left(I-\Pi_{n, m}\right) v\right\|_{\mathbb{V}} \leq D_{\Pi}\|v\|_{\mathbb{V}}, & \forall v \in \mathbb{V}, \\ \left\langle B w_{n}, v-\Pi_{n, m} v\right\rangle_{\mathbb{V}^{*}, \mathbb{V}}=0, & \forall w_{n} \in \mathbb{U}_{n}, \forall v \in \mathbb{V},\end{cases}
$$

where $I: \mathbb{V} \rightarrow \mathbb{V}$ is the identity map in $\mathbb{V}$. For simplicity, we write $\Pi$ instead of $\Pi_{n, m} .{ }^{17}$ For the existence of $\Pi$, note that the last identity (4.5c) requires that $\operatorname{dim} \mathbb{V}_{m} \geq$ $\operatorname{dim} \operatorname{Im}\left(\left.B\right|_{\mathbb{U}_{n}}\right)=\operatorname{dim} \mathbb{U}_{n}$ (for a bounded-below operator $B$ ). Schaback [38, Theorem 3] essentially guarantees the existence of $\Pi$ for sufficiently large $\mathbb{V}_{m}$ compared to $\mathbb{U}_{n}$, but it is unknown how much larger exactly $\mathbb{V}_{m}$ needs to be compared to $\mathbb{U}_{n}$ in the non-Hilbert Banach case. Note that (4.5a) implies (4.5b) with $D_{\Pi}=1+C_{\Pi}$, but to allow for sharper estimates, we prefer to retain the independent constant $D_{\Pi}$.

Theorem 4.5 (Discrete well-posedness). Consider the same hypotheses of Theorem 4.1. Let $M_{B}>0$ and $\gamma_{B}>0$ be as in (1.2). Let the finite-dimensional subspaces $\mathbb{U}_{n} \subset \mathbb{U}$ and $\mathbb{V}_{m} \subset \mathbb{V}$ satisfy Assumption 4.4.

(i) For any $f \in \mathbb{V}^{*}$, there exists a unique solution $\left(r_{m}, u_{n}\right) \in \mathbb{V}_{m} \times \mathbb{U}_{n}$ to discrete problem (1.8). ${ }^{18} 19$

(ii) Moreover, if $u \in \mathbb{U}$ is such that $B u=f$, then we have the a priori bounds:

$$
\left\{\begin{array}{l}
\left\|r_{m}\right\|_{\mathbb{V}} \leq\|f\|_{\mathbb{V}^{*}} \leq M_{B}\|u\|_{\mathbb{U}} \quad \text { and } \\
\left\|u_{n}\right\|_{\mathbb{U}} \leq \frac{C_{\Pi}}{\gamma_{B}}\left(1+C_{\mathrm{AO}}(\mathbb{V})\right)\|f\|_{\mathbb{V}^{*}} \leq \frac{C_{\Pi}}{\gamma_{B}}\left(1+C_{\mathrm{AO}}(\mathbb{V})\right) M_{B}\|u\|_{\mathbb{U}},
\end{array}\right.
$$

where $C_{\mathrm{AO}}(\mathbb{V})$ is the asymmetric-orthogonality constant of $\mathbb{V}$ (see Def. 3.9).

Proof. To prove existence, consider the equivalent discrete constrained minimization problem (4.4). The existence of a minimizer $r_{m} \in \mathbb{V}_{m} \cap\left(B \mathbb{U}_{n}\right)^{\perp}$ is guaranteed since the functional $v_{m} \mapsto \frac{1}{2}\left\|v_{m}\right\|_{\mathbb{V}}^{2}-\left\langle f, v_{m}\right\rangle_{\mathbb{V}^{*}, \mathbb{V}}$ is convex and continuous, and $\mathbb{V}_{m} \cap\left(B \mathbb{U}_{n}\right)^{\perp}$ is a closed subspace.

Next, we claim that there exists a $u_{n} \in \mathbb{U}_{n}$ such that

$$
\left\langle B u_{n}, v_{m}\right\rangle_{\mathbb{V}^{*}, \mathbb{V}}=\left\langle f-J_{\mathbb{V}}\left(r_{m}\right), v_{m}\right\rangle_{\mathbb{V}^{*}, \mathbb{V}}, \quad \forall v_{m} \in \mathbb{V}_{m} .
$$

To see this, consider the restricted operator $B_{n}: \mathbb{U}_{n} \rightarrow \mathbb{V}^{*}$ such that $B_{n} w_{n}=B w_{n}$, $\forall w_{n} \in \mathbb{U}_{n}$, and recall the injection $I_{m}: \mathbb{V}_{m} \rightarrow \mathbb{V}$. Then, the above translates into

$$
I_{m}^{*} B_{n} u_{n}=I_{m}^{*}\left(f-J_{\mathbb{V}}\left(r_{m}\right)\right) \quad \text { in }\left(\mathbb{V}_{m}\right)^{*} .
$$

\footnotetext{
${ }^{17}$ The Fortin condition is equivalent to the discrete inf-sup condition on $\left\{\left(\mathbb{U}_{n}, \mathbb{V}_{m}\right)\right\}$; see [25]. It classically appears in the study of mixed FEM [5, Section 5.4].

${ }^{18}$ Note that we do not require $\operatorname{Im}(B)=\mathbb{V}^{*}$. Indeed, for part (i), $f$ need not be in the $\operatorname{range} \operatorname{Im}(B)$.

${ }^{19}$ Assumption 4.4 is not needed for the existence of $\left(r_{m}, u_{n}\right)$, nor the uniqueness of $r_{m}$.
} 
Thus, to prove existence of $u_{n}, I_{m}^{*}\left(f-J_{\mathbb{V}}\left(r_{m}\right)\right)$ needs to be in the (closed) range of $I_{m}^{*} B_{n}: \mathbb{U}_{n} \rightarrow\left(\mathbb{V}_{m}\right)^{*}$. Since $r_{m}$ is the minimizer of (4.4), we have

$$
0=\left\langle J_{\mathbb{V}}\left(r_{m}\right)-f, I_{m} v_{m}\right\rangle_{\mathbb{V} *, \mathbb{V}}=\left\langle I_{m}^{*}\left(J_{\mathbb{V}}\left(r_{m}\right)-f\right), v_{m}\right\rangle_{\left(\mathbb{V}_{m}\right)^{*}, \mathbb{V}_{m}},
$$

$\forall v_{m} \in \mathbb{V}_{m} \cap\left(B \mathbb{U}_{n}\right)^{\perp}=\operatorname{ker}\left(B_{n}^{*} I_{m}\right)$, i.e., $I_{m}^{*}\left(f-J_{\mathbb{V}}\left(r_{m}\right)\right) \in\left(\operatorname{ker}\left(B_{n}^{*} I_{m}\right)\right)^{\perp}=\operatorname{Im}\left(I_{m}^{*} B_{n}\right)$.

To prove uniqueness, assume to the contrary that $\left(u_{n}, r_{m}\right)$ and $\left(\tilde{u}_{n}, \tilde{r}_{m}\right)$ are two distinct solutions. Then, by subtraction, it is immediate to see that:

$$
\left\langle J_{\mathbb{V}}\left(r_{m}\right)-J_{\mathbb{V}}\left(\tilde{r}_{m}\right), r_{m}-\tilde{r}_{m}\right\rangle_{\mathbb{V} * \mathbb{V}}=0,
$$

which implies that $\tilde{r}_{m}=r_{m}$ by strict monotonicity of $J_{\mathbb{V}}$ (see (2.2)). Going back to (1.8a) we now obtain $\left\langle B\left(u_{n}-\tilde{u}_{n}\right), v_{m}\right\rangle_{\mathbb{V}^{*}, \mathbb{V}}=0$, for all $v_{m} \in \mathbb{V}_{m}$. Therefore, by the Fortin-operator property $(4.5 \mathrm{c})$,

$$
\left\langle B\left(u_{n}-\tilde{u}_{n}\right), v\right\rangle_{\mathbb{V}^{*}, \mathbb{V}}=\left\langle B\left(u_{n}-\tilde{u}_{n}\right), \Pi v\right\rangle_{\mathbb{V}^{*}, \mathbb{V}}=0, \quad \forall v \in \mathbb{V} .
$$

Thus, $B\left(u_{n}-\tilde{u}_{n}\right)=0$ which implies $u_{n}-\tilde{u}_{n}=0$ since $B$ is bounded below.

To prove the bound (4.6a), replace $v_{m}=r_{m}$ in (1.8a), and use (1.8b) together with the Cauchy-Schwartz inequality. For (4.6b), see Proposition 4.12 in Section 4.4.

Although $\mathbb{V}_{m}$ should be sufficiently large for stability, there is no need for it to be close to the entire $\mathbb{V}$. The following proposition essentially shows that the goal of $\mathbb{V}_{m}$ is to resolve the residual $r \in \mathbb{V}$ of the ideal MinRes formulation (1.3) (cf. [18]).

Proposition 4.6 (Optimal $\left.\mathbb{V}_{m}\right)$. Consider the same hypotheses of Theorem 4.5. Let $u_{n} \in \mathbb{U}_{n}$ be the solution of the ideal MiNRES problem (1.3), and let $r=J_{\mathbb{V}}^{-1}(f-$ $\left.B u_{n}\right)$. If $r \in \mathbb{V}_{m}$, then $\left(r, u_{n}\right)$ is also the unique solution to the inexact case (1.8).

Proof. Notice that $J_{\mathbb{V}}(r)=f-B u_{n}$, so in particular (1.8a) is satisfied by $\left(r, u_{n}\right) \in$ $\mathbb{V}_{m} \times \mathbb{U}_{n}$. Recalling (3.4), and using Lemma 3.16 with $\mathbb{M}=B \mathbb{U}_{n} \subset \mathbb{V}^{*}=\mathbb{Y}$, we get:

$$
\left\langle B w_{n}, r\right\rangle_{\mathbb{V}^{*}, \mathbb{V}}=\left\langle B w_{n}, J_{\mathbb{V}^{*}}\left(B u-B u_{n}\right)\right\rangle_{\mathbb{V}^{*}, \mathbb{V}}=0, \quad \forall w_{n} \in \mathbb{U}_{n},
$$

where we used that $J_{\mathbb{V}^{*}}=J_{\mathbb{V}}^{-1}$ (recall from Section 2). This verifies (1.8b).

4.3. Error analysis. We next present an error analysis for the inexact MinReS discretization (1.5). Since the method is fundamentally related to (discrete) residuals, the most straightforward error estimate is of a posteriori type. This estimate happens to coincide with the Hilbert case; see [10] and [15, Proposition 3.2]. Immediately after, an a priori error estimate follows naturally from the a posteriori estimate. The constant in the resulting a priori error estimate can however be improved by resorting to an alternative analysis technique, which we present in Section 4.4.

TheOREM 4.7 (A posteriori error estimate). Consider the same hypotheses of Theorem 4.5. Let $f=B u \in \mathbb{V}^{*}$ and let $\left(r_{m}, u_{n}\right) \in \mathbb{V}_{m} \times \mathbb{U}_{n}$ be the unique solution to (1.8). Then $u_{n}$ satisfies the following a posteriori error estimate:

$$
\left\|u-u_{n}\right\|_{\mathbb{U}} \leq \frac{1}{\gamma_{B}} \operatorname{osc}(f)+\frac{C_{\Pi}}{\gamma_{B}}\left\|r_{m}\right\|_{\mathbb{V}},
$$

where the data-oscillation term $\operatorname{osc}(f)$ and $\left\|r_{m}\right\|_{\mathbb{V}}$ satisfy:

$$
\begin{aligned}
\operatorname{osc}(f):=\sup _{v \in \mathbb{V}} \frac{\langle f, v-\Pi v\rangle}{\|v\|_{\mathbb{V}}} \leq M_{B} D_{\Pi} \inf _{w_{n} \in \mathbb{U}_{n}}\left\|u-w_{n}\right\|_{\mathbb{U}} \leq M_{B} D_{\Pi}\left\|u-u_{n}\right\|_{\mathbb{U}}, \\
\left\|r_{m}\right\|_{\mathbb{V}} \leq M_{B} \inf _{w_{n} \in \mathbb{U}_{n}}\left\|u-w_{n}\right\|_{\mathbb{U}} \quad \leq M_{B}\left\|u-u_{n}\right\|_{\mathbb{U}} .
\end{aligned}
$$


Proof. Using that $B$ is bounded from below, and that $B u=f$, we get:

(by (1.2)) $\quad\left\|u-u_{n}\right\|_{\mathbb{U}} \leq \frac{1}{\gamma_{B}}\left\|B u-B u_{n}\right\|_{\mathbb{V}^{*}}=\frac{1}{\gamma_{B}} \sup _{v \in \mathbb{V}} \frac{\left\langle f-B u_{n}, v-\Pi v+\Pi v\right\rangle_{\mathbb{V}^{*}, \mathbb{V}}}{\|v\|_{\mathbb{V}}}$

(by $(4.5 c))$

$$
\leq \frac{1}{\gamma_{B}} \sup _{v \in \mathbb{V}} \frac{\langle f, v-\Pi v\rangle_{\mathbb{V}^{*}, \mathbb{V}}}{\|v\|_{\mathbb{V}}}+\frac{1}{\gamma_{B}} \sup _{v \in \mathbb{V}} \frac{\left\langle f-B u_{n}, \Pi v\right\rangle_{\mathbb{V}^{*}, \mathbb{V}}}{\|v\|_{\mathbb{V}}}
$$

(by (4.5a), (1.8a)) $\leq \frac{1}{\gamma_{B}} \operatorname{osc}(f)+\frac{C_{\Pi}}{\gamma_{B}} \sup _{v \in V} \frac{\left\langle J_{\mathbb{V}}\left(r_{m}\right), \Pi v\right\rangle_{\mathbb{V}^{*}, \mathbb{V}}}{\|\Pi v\|_{\mathbb{V}}}$

(by $(2.1)$ )

$$
\leq \frac{1}{\gamma_{B}} \operatorname{osc}(f)+\frac{C_{\Pi}}{\gamma_{B}}\left\|J_{\mathbb{V}}\left(r_{m}\right)\right\|_{\mathbb{V}^{*}}=\frac{1}{\gamma_{B}} \operatorname{osc}(f)+\frac{C_{\Pi}}{\gamma_{B}}\left\|r_{m}\right\|_{\mathbb{V}} .
$$

Next, using $f=B u,(4.5 \mathrm{c}),(1.2)$ and (4.5b), observe that for all $w_{n} \in \mathbb{U}_{n}$,

$$
\operatorname{osc}(f)=\sup _{v \in \mathbb{V}} \frac{\left\langle B u-B w_{n}, v-\Pi v\right\rangle_{\mathbb{V} *, \mathbb{V}}}{\|v\|_{\mathbb{V}}} \leq M_{B} D_{\Pi}\left\|u-w_{n}\right\|_{\mathbb{U}},
$$

while noting that $\left\|r_{m}\right\|_{\mathbb{V}}=\left\langle J_{\mathbb{V}}\left(r_{m}\right), r_{m}\right\rangle_{\mathbb{V}^{*}, \mathbb{V}} /\left\|r_{m}\right\|_{\mathbb{V}}$ by (2.1), and using (1.8), we have

$$
\left\|r_{m}\right\|_{\mathbb{V}}=\frac{\left\langle f-B u_{n}, r_{m}\right\rangle_{\mathbb{V}, \mathbb{V}}}{\left\|r_{m}\right\|_{\mathbb{V}}}=\frac{\left\langle B u-B w_{n}, r_{m}\right\rangle_{\mathbb{V} * \mathbb{V}}}{\left\|r_{m}\right\|_{\mathbb{V}}} \leq M_{B}\left\|u-w_{n}\right\|_{\mathbb{U}} .
$$

Corollary 4.8 (A priori error estimate I). Under the same conditions of Theorem $4.7, u_{n}$ satisfies the following a priori error estimate:

$$
\left\|u-u_{n}\right\|_{\mathbb{U}} \leq C \inf _{w_{n} \in \mathbb{U}_{n}}\left\|u-w_{n}\right\|_{\mathbb{U}}, \quad \text { with } C=\frac{\left(D_{\Pi}+C_{\Pi}\right) M_{B}}{\gamma_{B}} .
$$

Remark 4.9 (Oscillation). In the context of finite-element approximations, the data-oscillation term osc $(f)$ can generally be expected to be of higher order than indicated by the upper bound in (4.8a); see discussion in [10].

Remark 4.10 (Ideal MinReS). If $\mathbb{V}_{m}=\mathbb{V}$, then $\operatorname{osc}(f)=0, D_{\Pi}=0$ and $C_{\Pi}=1$ (set $\Pi=I$ ), so that (4.9) holds with $C=\frac{M_{B}}{\gamma_{B}}$, which recovers the estimate in [27] for the ideal MinRes discretization.

4.4. Direct a priori error analysis. A direct a priori error analysis is possible for the inexact MinRes discretization, without going through the a posteriori error estimate. The benefit of the direct analysis is that the resulting estimate is sharper than given in (4.9), as it explicitly includes geometric constants for $\mathbb{U}$ and $\mathbb{V}$.

The direct analysis is based on the sequence of inequalities (formalized below):

$$
\left\|u-u_{n}\right\|_{\mathbb{U}} \leq\left\|I-P_{n}\right\|\left\|u-w_{n}\right\|_{\mathbb{U}} \leq C\left\|P_{n}\right\|\left\|u-w_{n}\right\|_{\mathbb{U}}, \quad \forall w_{n} \in \mathbb{U}_{n},
$$

where $I$ is the identity, $P_{n}$ is the projector defined below in Definition 4.11, and the norm $\|\cdot\|$ corresponds to the standard operator norm.

Definition 4.11 (Nonlinear PG projector). Under the conditions of Theorem 4.5, the (inexact) nonlinear Petrov-Galerkin projector is defined by the map

$$
P_{n}: \mathbb{U} \rightarrow \mathbb{U}_{n} \quad \text { such that } \quad P_{n}(u):=u_{n}
$$

with $u_{n}$ the second argument of the solution $\left(r_{m}, u_{n}\right)$ of (1.8) with input data $f=B u$. 
The next result establishes important properties of $P_{n}$, including a fundamental bound that depends on the geometric constant $C_{\mathrm{AO}}(\mathbb{V}) \in[0,1]$ (recall Definition 3.9).

Proposition 4.12 (Nonlinear PG projector properties).

(i) $P_{n}$ is a nontrivial projector: $0 \neq P_{n}=P_{n} \circ P_{n} \neq I$.

(ii) $P_{n}$ is homogeneous: $P_{n}(\lambda u)=\lambda P_{n}(u)$, for all $u \in \mathbb{U}$ and all $\lambda \in \mathbb{R}$.

(iii) $P_{n}$ is bounded, in particular, ${ }^{20}$

$$
\left\|P_{n}\right\|=\sup _{u \in \mathbb{U}} \frac{\left\|P_{n}(u)\right\|_{\mathbb{U}}}{\|u\|_{\mathbb{U}}} \leq \frac{C_{\Pi}}{\gamma_{B}}\left(1+C_{\mathrm{AO}}(\mathbb{V})\right) M_{B} .
$$

(iv) $P_{n}$ is distributive as follows: $P_{n}\left(u-P_{n}(w)\right)=P_{n}(u)-P_{n}(w)$, for all $u, w \in \mathbb{U}$.

(v) $P_{n}$ is a quasi-linear projector as defined in Lemma 3.3(iv).

Proof. See Section A.3.

Property (iv) is key to establishing the first inequality in (4.10), indeed, for $w_{n} \in \mathbb{U}_{n}$,

$$
\left\|u-P_{n}(u)\right\|_{\mathbb{U}}=\left\|u-w_{n}-P_{n}\left(u-w_{n}\right)\right\|_{\mathbb{U}} \leq\left\|I-P_{n}\right\|\left\|u-w_{n}\right\|_{\mathbb{U}} .
$$

On the other hand, the second inequality in (4.10) can be established through properties (i)-(iii) and (v), as they correspond to the four requirements for the abstract nonlinear projector $Q$ of Lemma 3.3. Hence, that Lemma immediately provides a bound for $\left\|I-P_{n}\right\|$ depending on the Banach-Mazur geometric constant $C_{\mathrm{BM}}(\mathbb{U})$ :

Corollary 4.13 (Nonlinear PG projector estimate). $I-P_{n}$ satisfies:

$$
\left\|I-P_{n}\right\| \leq C_{S}\left\|P_{n}\right\|, \quad \text { with } C_{S}:=\min \left\{1+\left\|P_{n}\right\|^{-1}, C_{\mathrm{BM}}(\mathbb{U})\right\} .
$$

In conclusion, by combining (4.12), (4.13) and (4.11), we obtain our main result:

TheOrem 4.14 (A priori error estimate II). Consider the same hypotheses of Theorem 4.5. Let $f=B u$ and let $\left(r_{m}, u_{n}\right) \in \mathbb{V}_{m} \times \mathbb{U}_{n}$ be the unique solution to (1.8). Then $u_{n}$ satisfies the following a priori error estimate:

$$
\left\|u-u_{n}\right\|_{\mathbb{U}} \leq C \inf _{w_{n} \in \mathbb{U}_{n}}\left\|u-w_{n}\right\|_{\mathbb{U}},
$$

with $C=\min \left\{\frac{C_{\Pi}}{\gamma_{B}}\left(1+C_{\mathrm{AO}}(\mathbb{V})\right) M_{B} C_{\mathrm{BM}}(\mathbb{U}), 1+\frac{C_{\Pi}}{\gamma_{B}}\left(1+C_{\mathrm{AO}}(\mathbb{V})\right) M_{B}\right\}$.

Remark 4.15 (DPG). If $\mathbb{U}, \mathbb{V}$ are Hilbert spaces, then $C_{\mathrm{BM}}(\mathbb{U})=1$ and $C_{\mathrm{AO}}(\mathbb{V})=$ 0 , hence Theorem 4.14 holds with $C=C_{\Pi} M_{B} / \gamma_{B}$, which recovers the DPG result [26].

Corollary 4.16 (Petrov-Galerkin). Consider the same hypotheses of Theorem 4.14. If $\operatorname{dim} \mathbb{V}_{m}=\operatorname{dim} \mathbb{U}_{n}$ or $r_{m}=0$, then a Petrov-Galerkin statement holds: $\left\langle B u_{n}, v_{m}\right\rangle_{\mathbb{V}^{*}, \mathbb{V}}=\left\langle f, v_{m}\right\rangle_{\mathbb{V}^{*}, \mathbb{V}}, \forall v_{m} \in \mathbb{V}_{m}$, and $u_{n}$ satisfies the a priori error estimate:

$$
\left\|u-u_{n}\right\|_{\mathbb{U}} \leq C \inf _{w_{n} \in \mathbb{U}_{n}}\left\|u-w_{n}\right\|_{\mathbb{U}}, \quad \text { with } C=\min \left\{\frac{C_{\Pi}}{\gamma_{B}} M_{B} C_{\mathrm{BM}}(\mathbb{U}), 1+\frac{C_{\Pi}}{\gamma_{B}} M_{B}\right\} .
$$

Proof. If $\operatorname{dim} \mathbb{V}_{m}=\operatorname{dim} \mathbb{U}_{n}$, then (1.8b) implies $r_{m}=0$ (under the Fortin condition), which in turn reduces (1.8a) to a Petrov-Galerkin statement. Eq. (A.5) in the proof of Proposition 4.12 implies the simpler bound $\left\|P_{n}\right\|_{\mathbb{U}} \leq \frac{C_{\Pi}}{\gamma_{B}} M_{B}$, instead of (4.11). Thus, combining this bound with (4.12) and (4.13) yields the error estimate.

\footnotetext{
${ }^{20}$ It is also possible to prove $\left\|P_{n}\right\| \leq \frac{C_{\Pi}}{\gamma_{B}} C_{\mathrm{BM}}\left(\left(\mathbb{V}_{m}\right)^{*}\right) M_{B}$, by using Proposition 3.5 (with $\mathbb{Y}=$ $\left.\left(\mathbb{V}_{m}\right)^{*}\right)$ instead of Proposition 3.17 in the proof in Section A.3.
} 


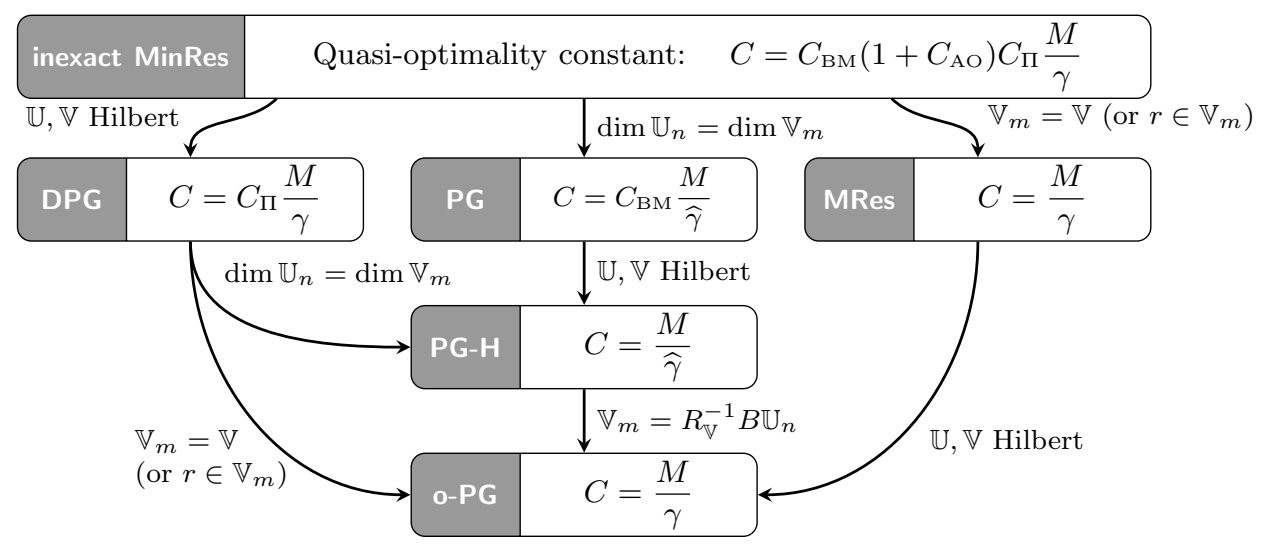

FIG. 2. Hierarchy of discretization methods, their connections, and their quasi-optimality constant $C$ in the a priori error estimate $\left\|u-u_{n}\right\|_{\mathbb{U}} \leq C \inf _{w_{n} \in \mathbb{U}_{n}}\left\|u-w_{n}\right\|_{\mathbb{U}}$. To lighten the notation, $\gamma \equiv \gamma_{B}, M \equiv M_{B}, C_{\mathrm{BM}} \equiv C_{\mathrm{BM}}(\mathbb{U}), C_{\mathrm{AO}} \equiv C_{\mathrm{AO}}(\mathbb{V})$. Furthermore, $\widehat{\gamma}$ is the discrete inf-sup constant in $P G$ methods, and $R_{\mathbb{V}}$ is the Riesz map in $\mathbb{V}$. Note that Theorem 4.14 has the complete result for $C=\min \{\cdot, \cdot\}$, while the figure only shows the non-trivial minimum. Legend: $\mathrm{PG}=$ PetrovGalerkin, PG-H = PG in Hilbert spaces, o-PG = optimal PG, DPG = discontinuous $\mathrm{PG}, \mathrm{MRes}=$ exact (or ideal) MINRES.

Remark 4.17 (Connections). The above error analysis unifies existing quasioptimality theories, because the inexact MinRES formulation directly encompasses the following more specialized methods: The exact (or ideal) MinRes method (see Remark 4.10 and Proposition 4.6), the inexact MinRes method in Hilbert spaces such as the DPG method (see Remark 4.15), and the Petrov-Galerkin method (see Corollary 4.16). Figure 2 shows how the various methods can be obtained from the general inexact MinRes formulation. It additionally shows further specialized methods: the PG method in Hilbert spaces and the optimal PG method (with ideal test space).

\section{Appendix A. Appendix: Proofs.}

A.1. Proof of Lemma 3.3. The inequality $\|I-Q\| \leq 1+\|Q\|=\left(1+\|Q\|^{-1}\right)\|Q\|$ is trivial, so we focus on proving

$$
\|y-Q(y)\|_{\mathbb{Y}} \leq C_{\mathrm{BM}}(\mathbb{Y})\|Q\|\|y\|_{\mathbb{Y}}, \quad \forall y \in \mathbb{Y} .
$$

If $y-Q(y)=0$, the result holds true immediately. If $Q(y)=0$ then, because requirement (i) implies $\|Q\| \geq 1$ and $C_{\mathrm{BM}}(\mathbb{Y}) \geq 1$ (recall Remark 3.2), we have

$$
\|y-Q(y)\|_{\mathbb{Y}}=\|y\|_{\mathbb{Y}} \leq C_{\mathrm{BM}}(\mathbb{Y})\|Q\|\|y\|_{\mathbb{Y}} .
$$

We can thus consider $y-Q(y) \neq 0$ and $Q(y) \neq 0$.

First observe that $y-Q(y)$ and $Q(y)$ are linearly independent. Indeed, suppose to the contrary that there exists $t \in \mathbb{R} \backslash\{0\}$ such that $y-Q(y)=t Q(y)$, then $y=(1+t) Q(y)$, hence applying $Q$ and using homogeneity (requirement (ii)), we get $t=0$ (a contradiction).

The proof follows using a 2-D geometrical argument. Define $\mathbb{W}:=\operatorname{span}\{Q(y)$, $y-Q(y)\}$, and note $\operatorname{dim} \mathbb{W}=2$. Let $T: \mathbb{W} \rightarrow \ell_{2}\left(\mathbb{R}^{2}\right)$ be any linear isomorphism. Set

$$
0 \neq \alpha:=\|T(y-Q(y))\|_{2} \quad \text { and } \quad 0 \neq \beta:=\|T Q(y)\|_{2},
$$


and subsequently, let $\tilde{y} \in \mathbb{W}$ be defined by

$$
\tilde{y}:=\frac{\alpha}{\beta} Q(y)+\frac{\beta}{\alpha}(y-Q(y)) .
$$

The proof will next be divided into four steps: (S1) shows that $\|y-Q(y)\|_{\mathbb{Y}} \leq$ $\left(\|T\|\left\|T^{-1}\right\|\right)\left\|\frac{\alpha}{\beta} Q(y)\right\|_{\mathbb{Y}} ;$ (S2) shows that $\left\|\frac{\alpha}{\beta} Q(y)\right\|_{\mathbb{Y}} \leq\|Q\|\|\tilde{y}\|_{\mathbb{Y}} ;$ (S3) shows that $\|\tilde{y}\|_{\mathbb{Y}} \leq\left(\|T\|\left\|T^{-1}\right\|\right)\|y\|_{\mathbb{Y}}$; and (S4) concludes that $\|y-Q(y)\|_{\mathbb{Y}} \leq C_{\mathrm{BM}}(\mathbb{Y})\|Q\|\|y\|_{\mathbb{Y}}$.

(S1) This follows from elementary arguments since $\beta \neq 0$ :

$$
\begin{aligned}
\|y-Q(y)\|_{\mathbb{Y}} & \leq\left\|T^{-1}\right\|\|T(y-Q(y))\|_{2} \\
& =\left\|T^{-1}\right\| \alpha \\
& =\left\|T^{-1}\right\| \frac{\alpha}{\beta}\|T Q(y)\|_{2} \leq\left\|T^{-1}\right\|\|T\|\left\|\frac{\alpha}{\beta} Q(y)\right\|_{\mathbb{Y}} .
\end{aligned}
$$

(S2) Use requirement (iv) with $\eta=\frac{\beta^{2}}{\alpha^{2}}$, and subsequently (ii) and (iii), to obtain:

$$
\left\|\frac{\alpha}{\beta} Q(y)\right\|_{\mathbb{Y}}=\left\|\frac{\alpha}{\beta} Q\left(Q(y)+\frac{\beta^{2}}{\alpha^{2}}(I-Q)(y)\right)\right\|_{\mathbb{Y}}=\|Q(\tilde{y})\|_{\mathbb{Y}} \leq\|Q\|\|\tilde{y}\|_{\mathbb{Y}} .
$$

(S3) The key point here is to observe that $\|T \tilde{y}\|_{2}=\|T y\|_{2}$, indeed,

(by (A.2) and (A.1))

$$
\|T \tilde{y}\|_{2}^{2}=\left\|\frac{\alpha}{\beta} T Q(y)+\frac{\beta}{\alpha} T(y-Q(y))\right\|_{2}^{2}
$$

$$
=\alpha^{2}+2 T Q(y) \cdot T(y-Q(y))+\beta^{2}=\|T(y-Q(y))+T Q(y)\|_{2}^{2}=\|T y\|_{2}^{2} .
$$

Therefore, $\|\tilde{y}\|_{\mathbb{Y}} \leq\left\|T^{-1}\right\|\|T \tilde{y}\|_{2}=\left\|T^{-1}\right\|\|T y\|_{2} \leq\left\|T^{-1}\right\|\|T\|\|y\|_{\mathbb{Y}}$.

(S4) Combining (S1)-(S3) we get $\|y-Q(y)\|_{\mathbb{Y}} \leq\left(\|T\|\left\|T^{-1}\right\|\right)^{2}\|Q\|\|y\|_{\mathbb{Y}}$. Finally, taking the infimum over all linear isomorphisms $T: \mathbb{W} \rightarrow \ell_{2}\left(\mathbb{R}^{2}\right)$ we obtain

$$
\|y-Q(y)\|_{\mathbb{Y}} \leq\left(d_{\mathrm{BM}}\left(\mathbb{W}, \ell_{2}\left(\mathbb{R}^{2}\right)\right)\right)^{2}\|Q\|\|y\|_{\mathbb{Y}} \leq C_{\mathrm{BM}}(\mathbb{Y})\|Q\|\|y\|_{\mathbb{Y}} .
$$

A.2. Proof of Lemma 3.12. Recall that $J_{\mathbb{Y}}$ and $J_{\mathbb{Y}^{*}}$ are single-valued bijections and $J_{\mathbb{Y}^{*}}=J_{\mathbb{Y}}^{-1}$. Property (i) is a direct consequence of the definition of $C_{\mathrm{AO}}(\mathbb{Y})$ (see (3.5)) and the fact that $J_{\mathbb{Y}}$ is single-valued.

To prove property (ii), we make use of property (i) replacing $\mathbb{Y}$ by $\mathbb{Y}^{*}$. We get

$$
C_{\mathrm{AO}}\left(\mathbb{Y}^{*}\right)=\sup _{\left(z^{*}, z_{0}^{*}\right) \in \mathcal{O}_{\mathbb{Y}^{*}}} \frac{\left\langle J_{\mathbb{Y}^{*}}\left(z^{*}\right), z_{0}^{*}\right\rangle_{\mathbb{Y}^{* *}, \mathbb{Y}^{*}}}{\left\|z_{0}^{*}\right\|_{\mathbb{Y}^{*}}\left\|z^{*}\right\|_{\mathbb{Y}^{*}}}=\sup _{\left(z^{*}, z_{0}^{*}\right) \in \mathcal{O}_{\mathbb{Y}^{*}}} \frac{\left\langle z_{0}^{*}, J_{\mathbb{Y}}^{-1}\left(z^{*}\right)\right\rangle_{\mathbb{Y}^{*}, \mathbb{Y}}}{\left\|z_{0}^{*}\right\|_{\mathbb{Y}^{*}}\left\|z^{*}\right\|_{\mathbb{Y}^{*}}} .
$$

Defining $z=J_{\mathbb{Y}}^{-1}\left(z^{*}\right)$ and $z_{0}=J_{\mathbb{Y}}^{-1}\left(z_{0}^{*}\right)$ we obtain

$$
\begin{aligned}
C_{\mathrm{AO}}\left(\mathbb{Y}^{*}\right) & =\sup _{\left(z^{*}, z_{0}^{*}\right) \in \mathcal{O}_{\mathbb{Y}^{*}}} \frac{\left\langle J_{\mathbb{Y}}\left(z_{0}\right), z\right\rangle_{\mathbb{Y}^{*}, \mathbb{Y}}}{\left\|z_{0}\right\|_{\mathbb{Y}}\|z\|_{\mathbb{Y}}}, \\
\text { with } \mathcal{O}_{\mathbb{Y}^{*}} & =\left\{\left(z^{*}, z_{0}^{*}\right) \in \mathbb{Y}^{*} \times \mathbb{Y}^{*}:\left\langle J_{\mathbb{Y}^{*}}\left(z_{0}^{*}\right), z^{*}\right\rangle_{\mathbb{Y}^{* *}, \mathbb{Y}^{*}}=0\right\} \\
& =\left\{\left(J_{\mathbb{Y}}(z), J_{\mathbb{Y}}\left(z_{0}\right)\right) \in \mathbb{Y}^{*} \times \mathbb{Y}^{*}:\left\langle J_{\mathbb{Y}}(z), z_{0}\right\rangle_{\mathbb{Y}^{*}, \mathbb{Y}}=0\right\} \\
& =\left\{\left(J_{\mathbb{Y}}(z), J_{\mathbb{Y}}\left(z_{0}\right)\right) \in \mathbb{Y}^{*} \times \mathbb{Y}^{*}:\left(z_{0}, z\right) \in \mathcal{O}_{\mathbb{Y}}\right\} .
\end{aligned}
$$

Hence the supremum in (A.3) can be taken over all $\left(z_{0}, z\right) \in \mathcal{O}_{\mathbb{Y}}$ which proves (ii). 
For the last property (iii) we make use of Corollary 2.4 to show that

$$
C_{\mathrm{AO}}(\mathbb{M})=\sup _{\left(z_{0}, z\right) \in \mathcal{O}_{\mathbb{M}}} \frac{\left\langle J_{\mathbb{M}}\left(z_{0}\right), z\right\rangle_{\mathbb{M}^{*}, \mathbb{M}}}{\|z\|_{\mathbb{Y}}\|\| z_{0} \|_{\mathbb{Y}}}=\sup _{\left(z_{0}, z\right) \in \mathcal{O}_{\mathbb{M}}} \frac{\left\langle J_{\mathbb{Y}}\left(I_{\mathbb{M}} z_{0}\right), I_{\mathbb{M}} z\right\rangle_{\mathbb{Y}^{*}, \mathbb{Y}}}{\|z\|_{\mathbb{Y}}\|\| z_{0} \|_{\mathbb{Y}}} .
$$

The fact $C_{\mathrm{AO}}(\mathbb{M}) \leq C_{\mathrm{AO}}(\mathbb{Y})$ follows by noting that the supremum in $C_{\mathrm{AO}}(\mathbb{Y})$ is over a larger set (i.e., $\left.I_{\mathbb{M}} \mathcal{O}_{\mathbb{M}} \subset \mathcal{O}_{\mathbb{Y}}\right)$. Indeed, if $\left(z_{0}, z\right) \in \mathcal{O}_{\mathbb{M}}$, then $\left(I_{\mathbb{M}} z_{0}, I_{\mathbb{M}} z\right) \in \mathbb{Y} \times \mathbb{Y}$ and

$$
\left\langle J_{\mathbb{Y}}\left(I_{\mathbb{M}} z\right), I_{\mathbb{M}} z_{0}\right\rangle_{\mathbb{Y}^{*}, \mathbb{Y}}=\left\langle J_{\mathbb{M}}(z), z_{0}\right\rangle_{\mathbb{M}^{*}, \mathbb{M}}=0,
$$

by Corollary 2.4. Hence $\left(I_{\mathbb{M}} z_{0}, I_{\mathbb{M}} z\right) \in \mathcal{O}_{\mathbb{Y}}$. The last inequality combined with (ii) implies (iii) because $C_{\mathrm{AO}}(\mathbb{Y})=C_{\mathrm{AO}}\left(\mathbb{Y}^{*}\right) \leq C_{\mathrm{AO}}\left(\mathbb{M}^{*}\right)=C_{\mathrm{AO}}(\mathbb{M}) \leq C_{\mathrm{AO}}(\mathbb{Y})$.

A.3. Proof of Proposition 4.12. We proceed item by item.

(i) Take $u \in \mathbb{U}, u_{n}=P_{n}(u)$, and substitute $f=B u_{n}$ in (1.8a). Then the unique solution of $(1.8)$ is $\left(0, u_{n}\right)$. Therefore $P_{n}\left(P_{n}(u)\right)=P_{n}\left(u_{n}\right)=u_{n}$. The fact that $P_{n} \neq 0$ and $P_{n} \neq I$ is easy to verify whenever $\mathbb{U}_{n} \neq\{0\}$ and $\mathbb{U}_{n} \neq \mathbb{U}$.

(ii) The result follows by multiplying both equations of the mixed system (1.8) by $\lambda \in \mathbb{R}$ and using the homogeneity of the duality map (recall from Section 2).

(iii) Set $f=B u$ and let $\left(r_{m}, u_{n}\right) \in \mathbb{V}_{m} \times \mathbb{U}_{n}$ denote the solution to (1.8). Then

$$
\left\|P_{n}(u)\right\|_{\mathbb{U}}=\left\|u_{n}\right\|_{\mathbb{U}} \leq \frac{1}{\gamma_{B}} \sup _{v \in \mathbb{V}} \frac{\left\langle B u_{n}, v\right\rangle_{\mathbb{V} * \mathbb{V}}}{\|v\|_{\mathbb{V}}} \leq \frac{C_{\Pi}}{\gamma_{B}} \sup _{v \in \mathbb{V}} \frac{\left\langle B u_{n}, \Pi v\right\rangle_{\mathbb{V}^{*}, \mathbb{V}}}{\|\Pi v\|_{\mathbb{V}}}
$$

Let $y_{m}=I_{m} J_{\mathbb{V}_{m}}^{-1}\left(I_{m}^{*} B u_{n}\right)$ and note that $y_{m} \in \mathbb{V}_{m} \subset \mathbb{V}$ is the supremizer of the last expression in (A.4). Hence, using (1.8a) we get

$$
\begin{aligned}
\left\|P_{n}(u)\right\|_{\mathbb{U}} & \leq \frac{C_{\Pi}}{\gamma_{B}} \frac{\left\langle B u_{n}, y_{m}\right\rangle_{\mathbb{V}^{*}, \mathbb{V}}}{\left\|y_{m}\right\|_{\mathbb{V}}} \\
& =\frac{C_{\Pi}}{\gamma_{B}}\left(\frac{\left\langle B u, y_{m}\right\rangle_{\mathbb{V} * \mathbb{V}}}{\left\|y_{m}\right\|_{\mathbb{V}}}-\frac{\left\langle J_{\mathbb{V}}\left(r_{m}\right), y_{m}\right\rangle_{\mathbb{V}^{*}, \mathbb{V}}}{\left\|r_{m}\right\|_{\mathbb{V}}\left\|y_{m}\right\|_{\mathbb{V}}}\left\|r_{m}\right\|_{\mathbb{V}}\right) .
\end{aligned}
$$

The first term in brackets is $\leq M_{B}\|u\|_{\mathbb{U}}$. To bound the second term, note

$$
\left\langle J_{\mathbb{V}}\left(y_{m}\right), r_{m}\right\rangle_{\mathbb{V}^{*}, \mathbb{V}}=\left\langle B u_{n}, r_{m}\right\rangle_{\mathbb{V}^{*}, \mathbb{V}}=0
$$

where we used (1.8b). Thus, $\left(r_{m}, y_{m}\right) \in \mathcal{O}_{\mathbb{V}}$ (see Lemma 3.12) which implies that the second term is bounded by $C_{\mathrm{AO}}(\mathbb{V})\left\|r_{m}\right\|_{\mathbb{V}}$. Using (4.6a), we get

$$
\left\|P_{n}(u)\right\|_{\mathbb{U}} \leq \frac{C_{\Pi}}{\gamma_{B}}\left(1+C_{\mathrm{AO}}(\mathbb{V})\right) M_{B}\|u\|_{\mathbb{U}} . \quad \forall u \in \mathbb{U}
$$

We note that an alternative proof can be given based on Proposition 3.17 (with $\left.\mathbb{Y}=\left(\mathbb{V}_{m}\right)^{*}\right)$ and Lemma 3.12.

(iv) Let $\left(r_{m}, u_{n}\right)$ be the solution of the mixed system (1.8) and for some $\widetilde{w} \in \mathbb{U}$, let $\widetilde{w}_{n}=P_{n}(\widetilde{w}) \in \mathbb{U}_{n}$. By subtracting $\left\langle B \widetilde{w}_{n}, v_{m}\right\rangle_{\mathbb{V} *, \mathbb{V}}$ on both sides of (1.8), we get that $\left(r_{m}, u_{n}-\widetilde{w}_{n}\right)$ is the unique solution of (1.8) with right-hand side $\left\langle B\left(u-\widetilde{w}_{n}\right), v_{m}\right\rangle_{\mathbb{V} *, \mathbb{V}}$. Therefore

$$
P\left(u-\widetilde{w}_{n}\right)=u_{n}-\widetilde{w}_{n} .
$$


(v) Statement (v) follows from statements (ii) and (iv). Indeed, for any $\eta \in \mathbb{R}$,

$$
\begin{array}{rlrl}
\text { (by (ii)) } & P_{n}\left(P_{n}(u)+\eta\left(u-P_{n}(u)\right)\right) & =P_{n}\left(\eta u+P_{n}((1-\eta) u)\right) \\
& =P_{n}(\eta u)+P_{n}((1-\eta) u) \\
\text { (by (iv) }) & & =P_{n}(u) .
\end{array}
$$

Acknowledgments. IM and KvdZ are grateful to Leszek Demkowicz for his early encouragement to investigate a Banach-space theory of DPG, and to Jay Gopalakrishnan for insightful conversations. KvdZ is also thankful to Michael Holst and Sarah Pollock for initial discussions on the topic, and to Weifeng Qiu, Paul Houston and Sarah Roggendorf for additional discussions. IM and KvdZ thank the anonymous reviewers for their helpful comments and suggestions (one of which led to the perceptive Corollary 2.4).

\section{REFERENCES}

[1] I. BABUŠKA, Error-bounds for finite element method, Numer. Math., 16 (1971), pp. 322-333.

[2] F. Bertrand, L. Demkowicz, J. Gopalakrishnan, and N. Heuer, Recent advances in leastsquares and discontinuous Petrov-Galerkin finite element methods, Comput. Methods Appl. Math., 19 (2019), pp. 395-397.

[3] M. Billaud-Friess, A. Nouy, And O. ZAhm, A tensor approximation method based on ideal minimal residual formulations for the solution of high-dimensional problems, M2AN Math. Model. Numer. Anal., 48 (2014), pp. 1777-1806.

[4] P. B. Bochev And M. D. Gunzburger, Least-Squares Finite Element Methods, vol. 166 of Applied Mathematical Sciences, Springer Science \& Business Media, 2009.

[5] D. Boffi, F. Brezzi, And M. Fortin, Mixed Finite Element Methods and Applications, vol. 44 of Springer Series in Computational Mathematics, Springer, Berlin, 2013.

[6] D. Braess, Nonlinear Approximation Theory, vol. 7 of Springer Series in Computational Mathematics, Springer, Berlin, 1986.

[7] H. Brezis, Functional Analysis, Sobolev Spaces and Partial Differential Equations, Universitext, Springer, New York, 2011.

[8] P. Cantin And N. Heuer, A DPG framework for strongly monotone operators, SIAM J. Numer. Anal., 56 (2018), pp. 2731-2750.

[9] C. Carstensen, P. Bringmann, F. Hellwig, and P. Wriggers, Nonlinear discontinuous Petrov-Galerkin methods, Numer. Math., 139 (2018), pp. 529-561.

[10] C. Carstensen, L. Demkowicz, and J. Gopalakrishnan, A posteriori error control for DPG methods, SIAM J. Numer. Anal., 52 (2014), pp. 1335-1353.

[11] J. Chan, L. Demkowicz, AND R. Moser, A DPG method for steady viscous compressible flow, Comput. \& Fluids, 98 (2014), pp. 69-90.

[12] C. Chidume, Geometric Properties of Banach Spaces and Nonlinear Iterations, vol. 1965 of Lecture Notes in Mathematics, Springer, London, 2009.

[13] P. G. CiARLet, Linear and Nonlinear Functional Analysis with Applications, SIAM, Philadelphia, 2013.

[14] I. Cioranescu, Geometry of Banach Spaces, Duality Mappings and Nonlinear Problems, vol. 62 of Mathematics and Its Applications, Kluwer Academic Publishers, Dordrecht, The Netherlands, 1990.

[15] A. Cohen, W. Dahmen, And G. Welper, Adaptivity and variational stabilization for convection-diffusion equations, M2AN Math. Model. Numer. Anal., 46 (2012), pp. 12471273.

[16] W. Dahmen, C. Huang, C. Schwab, and G. Welper, Adaptive Petrov-Galerkin methods for first order transport equations, SIAM J. Numer. Anal., 50 (2012), pp. 2420-2445.

[17] K. Deimling, Nonlinear Functional Analysis, Springer, Berlin, 1985.

[18] L. Demkowicz, T. Führer, N. Heuer, and X. Tian, The double adaptivity paradigm (how to circumvent the discrete inf-sup conditions of Babuška and Brezzi), Tech. Report 10-07, Institute for Computational Engineering and Sciences (ICES), The University of Texas at Austin, Austin, Texas, USA, 2019.

[19] L. Demkowicz and J. Gopalakrishnan, An overview of the discontinuous Petrov Galerkin method, in Recent Developments in Discontinuous Galerkin Finite Element Methods 
for Partial Differential Equations: 2012 John H Barrett Memorial Lectures, X. Feng, O. Karakashian, and Y. Xing, eds., vol. 157 of The IMA Volumes in Mathematics and its Applications, Springer, Cham, 2014, pp. 149-180.

[20] L. Demkowicz and J. Gopalakrishnan, Discontinuous Petrov-Galerkin (DPG) method, in Encyclopedia of Computational Mechanics, Second Edition, E. Stein, R. de Borst, and T. J. R. Hughes, eds., Wiley, 2017. Part 2 Fundamentals.

[21] L. Demkowicz and P. Zanotti, Construction of DPG Fortin operators revisited, Comput. Math. Appl., (2020). https://doi.org/10.1016/j.camwa.2020.07.020.

[22] V. Eijkhout and P. Vassilevski, The role of the strengthened Cauchy-Buniakowskii-Schwarz inequality in multilevel methods, SIAM Rev., (1991), pp. 405-419.

[23] I. Ekeland and R. TÉmam, Convex Analysis and Variational Problems, North-Holland Publishing Company, Amsterdam, 1976.

[24] A. Ern And J.-L. Guermond, Theory and Practice of Finite Element Methods, vol. 159 of Applied Mathematical Sciences, Springer-Verlag, New York, 2004.

[25] A. Ern and J.-L. Guermond, A converse to Fortin's Lemma in Banach spaces, C. R. Math. Acad. Sci. Paris, 354 (2016), pp. 1092-1095.

[26] J. Gopalakrishnan and W. QIU, An analysis of the practical DPG method, Math. Comp., 83 (2014), pp. 537-552.

[27] J. L. Guermond, A finite element technique for solving first-order PDEs in $L^{p}$, SIAM J. Numer. Anal., 42 (2004), pp. 714-737.

[28] P. Houston, I. Muga, S. Roggendorf, and K. G. van der Zee, The Convection-DiffusionReaction Equation in Non-Hilbert Sobolev Spaces: A Direct Proof of the Inf-Sup Condition and Stability of Galerkin's Method, Comput. Methods Appl. Math., 19 (2019), pp. 503-522.

[29] P. Houston, S. Roggendorf, and K. G. van der Zee, Eliminating Gibbs phenomena: A nonlinear Petrov-Galerkin method for the convection-diffusion-reaction equation, Comput. Math. Appl., 80 (2020), pp. 851-873.

[30] D. JeRison and C. E. Kenig, The inhomogeneous Dirichlet problem in Lipschitz domains, J. Funct. Anal., 130 (1995), pp. 161-219.

[31] W. B. Johnson and J. Lindenstrauss, Basic concepts in the geometry of Banach spaces, in Handbook of the Geometry of Banach Spaces, W. B. Johnson and J. Lindenstrauss, eds., vol. 1, Elsevier Science B. V., 2001, ch. 1, pp. 1-84.

[32] T. Kato, Estimation of iterated matrices with application to von Neumann condition, Numer. Math., 2 (1960), pp. 22-29.

[33] J. Li And L. Demkowicz, An $L^{p}$-DPG method for the convection-diffusion problem, Comput. Math. Appl., (2020). https://doi.org/10.1016/j.camwa.2020.08.013.

[34] J. Lions, Quelques Méthodes de Résolution des Problèmes aux Limites Non Linéaires, Études Mathématiques, Dunod, 1969.

[35] F. Millar, I. Muga, and K. G. Van Der Zee, Projection in negative norms and the regularization of rough linear functionals, 2020. In preparation.

[36] I. Muga, M. J. W. Tyler, And K. G. van Der Zee, The Discrete-Dual Minimal-Residual Method (DDMRes) for Weak Advection-Reaction Problems in Banach Spaces, Comput. Methods Appl. Math., 19 (2019), pp. 557-579.

[37] J. T. Oden And L. F. Demkowicz, Applied Functional Analysis, CRC Press, 2nd ed., 2010.

[38] R. Sснаваск, All well-posed problems have uniformly stable and convergent discretizations, Numer. Math., 132 (2016), pp. 597-630.

[39] I. Singer, Best Approximation in Normed Linear Spaces by Elements of Linear Subspaces, vol. 171 of Die Grundlehren der mathematischen Wissenshaften, Springer, Berlin, 1970.

[40] I. Stakgold and M. Holst, Green's Functions and Boundary Value Problems, vol. 99 of Pure and Applied Mathematics, John Wiley \& Sons, Hoboken, New Jersey, $3^{\text {rd }}$ ed., 2011.

[41] A. Stern, Banach space projections and Petrov-Galerkin estimates, Numer. Math., 130 (2015), pp. $125-133$.

[42] D. SzYLD, The many proofs of an identity on the norm of oblique projections, Numer. Algorithms, 42 (2006), pp. 309-323.

[43] P. WojtaszczyK, Banach Spaces for Analysts, no. 25 in Cambridge studies for advanced mathematics, Cambridge University Press, Cambridge, 1991.

[44] J. Xu And L. Zikatanov, Some observations on Babuška and Brezzi theories, Numer. Math., 94 (2003), pp. 195-202.

[45] E. ZEIDLER, Nonlinear Functional Analysis and its Applications, II/B: Nonlinear Monotone Operators, Springer-Verlag, New York, 1990.

[46] J. Zitelli, I. Muga, L. Demkowicz, J. Gopalakrishnan, D. Pardo, and V. M. Calo, A class of discontinuous Petrov-Galerkin methods. Part IV: The optimal test norm and time-harmonic wave propagation in 1D, J. Comput. Phys., 230 (2011), pp. 2406-2432. 